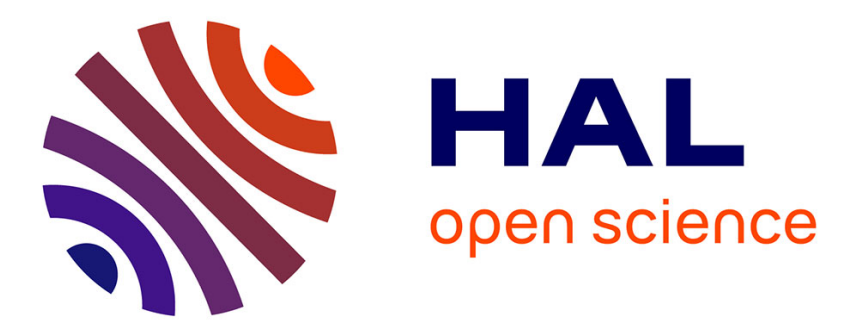

\title{
Mettre en concurrence ses fournisseurs-partenaires
}

Thomas Reverdy

\section{To cite this version:}

Thomas Reverdy. Mettre en concurrence ses fournisseurs-partenaires: Comment les acheteurs reconfigurent les échanges interindustriels. 2009. halshs-00484103

\section{HAL Id: halshs-00484103 \\ https://shs.hal.science/halshs-00484103}

Preprint submitted on 17 May 2010

HAL is a multi-disciplinary open access archive for the deposit and dissemination of scientific research documents, whether they are published or not. The documents may come from teaching and research institutions in France or abroad, or from public or private research centers.
L'archive ouverte pluridisciplinaire HAL, est destinée au dépôt et à la diffusion de documents scientifiques de niveau recherche, publiés ou non, émanant des établissements d'enseignement et de recherche français ou étrangers, des laboratoires publics ou privés. 


\title{
Mettre en concurrence ses fournisseurs-partenaires
}

\section{Comment les acheteurs reconfigurent les échanges interindustriels}

\author{
Thomas Reverdy
}

\begin{abstract}
Résumé
Dans leurs relations avec leurs fournisseurs, les clients industriels articulent coopération et compétition: la coopération répond aux besoins d'ajustements réciproques des systèmes de production, de partage des savoirs techniques, de dynamiques communes de résolution de problèmes; la compétition contribue à la maîtrise des prix. Huit cas d'interventions de la fonction Achats ont été analysés dans des situations où les fournisseurs sont fortement impliqués dans une collaboration avec leur client. En suivant la fonction Achat, notre recherche explicite comment celle-ci organise l'interchangeabilité des fournisseurs : il s'agit de réduire la singularité des besoins internes et de préciser leur formalisation, mais aussi d'agir sur les fournisseurs de façon à ce qu'ils proposent une offre et un niveau de service comparable entre eux. C'est ainsi que l'acheteur tente de rééquilibrer la relation de pouvoir en faveur du client. L'article s'interroge particulièrement sur les conditions de l'adhésion des clients internes et des fournisseurs à cette démarche : comment ne pas décourager l'investissement des fournisseurs dans la relation de service dont le client interne est dépendant? La mise en concurrence des « fournisseurs-partenaires » s'apparente donc à une construction stratégique et politique, tant à l'intérieur de l'entreprise cliente et qu'à l'intérieur du réseau de fournisseurs.
\end{abstract}


La libéralisation des échanges économiques et les nouvelles technologies de l'information offrent aux donneurs d'ordre industriels la possibilité de mettre en concurrence leurs fournisseurs existants avec des fournisseurs outsiders issus du monde entier (Friedman, 2005). Selon de nombreux observateurs (Berger, 2006), cette dynamique de mondialisation est modérée par les besoins de coordination des activités industrielles, qui plaident pour une proximité territoriale des activités et pour des engagements durables entre donneurs d'ordre et sous-traitants. Cette seconde thèse fait écho à de nombreuses recherches qui montrent que des relations durables entre un nombre limité de partenaires, permettent de réduire les incertitudes qui pèsent sur la qualité des échanges (Podolny, 1994, Dyer, 1997, Comet, 2005) et peuvent faciliter des investissements spécifiques, la recherche de synergie, les apprentissages communs et l'innovation (Powell, 1990, 1996, Ranjay, Gargiulo, 1999, Dyer, Nobeoka, 2000, Holm et al. 1999, Uzzi, 1997).

Dans un contexte d'accélération de la mondialisation, nous avons besoin de mieux connaître les processus par lesquels les configurations d'échange et les modalités de coordination évoluent. Notre recherche contribue à cette question en étudiant comment les donneurs d'ordre industriels procèdent pour ajuster la configuration des fournisseurs et pour «régler» leur niveau d'engagement.

Dans la perspective de l'analyse stratégique (Friedberg, 1993), nous considérons qu'une relation de coopération n'empêche pas l'action stratégique des acteurs, l'exercice d'un certain opportunisme (Neuville, 1998) ou l'existence de relations de dépendance asymétriques. Ces relations de dépendance tiennent aux différentes ressources pertinentes, aux actifs spécifiques engagés, mais aussi à la configuration des acteurs (Lazega, Mounier 2002), par exemple, l'existence d'alternatives. Ainsi, dans le cadre de relations coopératives, le client dispose d'une diversité de stratégie pour renforcer son pouvoir: chercher à réduire sa dépendance à des ressources extérieures, réduire les actifs spécifiques (Williamson, 1986) ou intervenir sur la configuration des acteurs qui participent (effectivement ou potentiellement) à l'échange en mobilisant un plus large réseau de fournisseurs...

Notre recherche s'intéresse à des relations entre clients et fournisseurs où ces derniers investissent particulièrement dans la « relation de service » (De Bandt, Gadrey, 1997) avec leur client. C'est généralement le cas dans la sous-traitance, dans les prestations de services à l'industrie, ou dans les projets d'équipements industriels spécialisés. L'investissement des fournisseurs comprend à la fois une forte singularisation de l'offre et de nombreux ajustements non formalisés pour s'articuler avec le système sociotechnique du client, tant en termes de 
performance technique, d'utilisation, d'organisation, de flux... L'accent est mis sur la satisfaction du client, quitte à aller au-delà de ce que prévoit le contrat. La «relation de service » secrète de nombreuses interdépendances opérationnelles (sans que celles-ci soient suffisamment importantes pour empêcher toute sortie de la relation). Notre recherche investigue plus précisément l'organisation de la concurrence entre des fournisseurs en place et de nouveaux fournisseurs pour le renouvellement de contrats ou de nouveaux projets.

Notre stratégie de recherche consiste à entrer à l'intérieur des entreprises clientes pour identifier les acteurs impliqués (Pettigrew, 1975) et plus précisément à nous intéresser à l'action de la fonction Achats, qui a explicitement pour mission de «susciter chez les fournisseurs un esprit de compétitivité créative, de promouvoir les propositions des fournisseurs et leurs suggestions pour la réduction des coûts et/ou l'amélioration des performances»(AFNOR, 1990). Cette fonction connait un important développement, qui se traduit par des recrutements significatifs, des rémunérations croissantes, un domaine d'intervention qui couvre toutes les catégories d'achat, des matières premières aux prestations intellectuelles. A ce titre, les acheteurs sont devenus les principaux «professionnels du marché» (Cochoy, Dubuisson-Quellier, 2000) dans le monde industriel.

Notre hypothèse de recherche était la suivante : les acheteurs tiennent un rôle d'intermédiaire entre les fournisseurs et le reste de l'entreprise cliente, ou plus précisément les «clients internes » concernés par l'activité des fournisseurs : directions industrielles, ligne de production, bureau d'étude... L'action des acheteurs consiste donc principalement à intervenir sur la configuration des échanges stratégiques entre clients internes et fournisseurs en place ou alternatifs. Leur action prend place dans un système d'action, sur lequel ils vont tenter d'agir : les clients internes sont les premiers bénéficiaires des investissements relationnels des fournisseurs (Neuville, 1998). Les acheteurs sont dépendants des clients internes : ce sont ceux qui apprécient la qualité technique des offres.

Autrement dit, pour comprendre comment s'articule compétition et coopération, il s'agit donc d'étudier les stratégies et les pratiques par lesquelles les acheteurs parviennent à s'interposer entre les fournisseurs et les clients internes, lesquels pourraient avoir une préférence pour des relations directes et stables.

Cet article est organisé de la façon suivante : une première partie explicite le débat en sociologie économique sur concurrence et coopération dans les relations entre clients et fournisseurs et identifie les principaux résultats des travaux existants. Une deuxième partie explicite notre stratégie de recherche, fondée sur l'analyse de huit situations de remise en question de relation 
partenariale, l'enquête auprès des différentes parties prenantes, la formalisation de ces situations et la comparaison.

Nous présentons ensuite les résultats de notre recherche. Nous discutons d'abord la mission confiée aux acheteurs : repenser les marchés amont afin d'intensifier la compétition. Les deux parties suivantes se concentrent sur le travail organisationnel de l'acheteur, sur la façon dont il reconfigure ces relations de coopération: la rationalisation des flux opérationnels entre fournisseurs et client, la mobilisation de fournisseurs alternatifs. Enfin les deux dernières parties s'interrogent sur les conditions de l'adhésion des fournisseurs et des clients internes aux différentes démarches de l'acheteur. Une conclusion revient sur les apports de notre recherche, ses limites et les nouvelles pistes de questionnement qu'elle propose.

\section{Questionnement de recherche : les réseaux à l'épreuve du marché}

Nous nous appuyons sur une définition étendue du marché : l'organisation par les clients d'une « compétition entre parties pour une opportunité d'échange (Max Weber, 1922, Swedberg, 1994, François 2004). Pour la sociologie des marchés (François, 2008), ce qui prime dans l'analyse, c'est la configuration des acteurs qui échangent et la nature de leurs échanges. Mettre en concurrence signifie donc d'intervenir sur le contenu des échanges et sur la configuration des acteurs, de façon à ce que plusieurs offreurs participent à une «lutte pacifique » pour accéder à l'échange.

\section{Configuration du marché et nature des échanges}

Il existe en sociologie économique un ensemble de travaux convergents qui établissent des liens entre la nature des échanges et la configuration des acteurs dans laquelle s'exercent les échanges. Ainsi, quand la nature de ce qui est échangé ne peut pas être spécifiée a priori (incertitudes sociotechniques, incertitudes sur la fiabilité du partenaire, échanges informels de connaissances...) ces travaux observent que la stabilité et l'exclusivité des relations s'accroissent. Ce résultat est validé dans des activités aussi diverses que les banques d'investissement (Poldony, 1994), que les activités industrielles (Dyer, 1997) ou artisanales (Comet, 2007, Uzzi 1997), que la recherche et développement en biotechnologie (Powell et al., 1996). De même, la stabilité et l'exclusivité des relations s'accroissent quand le contenu des échanges est spécifique au fournisseur et au client. Les stratégies de différenciation des fournisseurs permet la constitution de niches où l'intensité concurrentielle est limitée (White, 1981).

Ces résultats doivent être mis en perspective avec le travail de Granovetter (1973) qui montre que la diversification des liens permet d'accéder à de meilleures informations et de meilleures opportunités que des liens exclusifs. Les configurations peuvent évoluer avec la maturité du 
marché (Baker et al., 1998) qui peut entrainer une diversité de relations, certaines exclusives, d'autres très compétitives. Uzzi (1999) suggère que les clients et fournisseurs peuvent constituer un portefeuille de relations équilibré entre deux formes : des relations où le principal mécanisme de coordination est la concurrence et des relations où les principaux mécanismes de coordination sont la réciprocité, la réputation, les engagements formels.

L'analyse des configurations des acteurs qui contribuent aux échanges peut se compléter d'une analyse plus fine des interdépendances au sein de chacune des relations (Lazega, Mounier, 2002). La configuration tient un rôle important dans les relations d'interdépendance: Burt (1992) nous rappelle que l'on est d'autant plus dépendant d'un partenaire quand l'on n'a pas de partenaire alternatif avec qui échanger. Mais l'étude de la configuration seule ne suffit pas à caractériser les relations de dépendance. On peut avoir plusieurs partenaires potentiels alternatifs, mais être dépendant de l'un d'entre eux au point de ne pas parvenir réellement à l'abandonner... Bref, les jeux de pouvoir entre clients et fournisseurs portent autant sur les configurations que sur les relations.

Ainsi, plusieurs travaux de recherche sur les relations clients fournisseurs se focalisent sur les interdépendances, la combinaison entre compétition et coopération, avec un intérêt plus limité pour les configurations d'acteurs. Une enquête réalisée par Gulati et Sytch (2007) auprès des acheteurs de constructeurs automobile montre que ces derniers apprécient positivement les « actions communes » (les efforts d'amélioration de la qualité, de contrôle des coûts, les propositions de modification...) mais combattent toutes situations où ils n'auraient pas d'alternative, qu'elles soient dues à des coûts de changement de fournisseur trop importants, une compétition insuffisante, l'absence d'alternative à court ou moyen terme. S'appuyant sur une enquête auprès des constructeurs et des sous-traitants, Whitford (2005) confirme ce résultat : les donneurs d'ordre adoptent une attitude contradictoire ; ils exigent une collaboration intense aux projets de nouveaux véhicules et maintiennent le jeu concurrentiel pour exercer une pression permanente sur les prix ${ }^{1}$. La menace d'acheter les mêmes produits à des fournisseurs issus de pays à bas coûts de main d'œuvre permet de peser sur les prix. Ainsi, l'attitude collaborative du fournisseur n'implique pas nécessairement un engagement réciproque du client.

\footnotetext{
${ }^{1}$ Ces travaux remettent en question la thèse de Powell (1996) qui considère que la concurrence (le «marché ») et la coopération (le « réseau ») constituent deux formes de coordinations quasiment antinomiques : chacun repose sur un climat relationnel tout à fait spécifique, ouvert et confiance pour le premier, opportuniste et suspicieux pour le second. Difficile d'imaginer une relation où les deux climats coexistent.
} 
Si on résume ces différents travaux, on peut formuler l'hypothèse suivante : si des relations durables entre partenaires permettent de réduire les incertitudes sur la qualité de la relation et d'encourager des « actions communes » (Gulati, Sytch, 2007), la compétition entre fournisseurs (autrement dit, la menace qu'un autre fournisseur se substitue totalement ou partiellement au fournisseur en place) en est rarement absente. Whitford (2005) avance même que les situations où la compétition est absente (remplacée par la confiance ou la réputation seules) sont l'exception. La compétition tend à s'intensifier avec la maturité de l'activité et les opportunités offertes par la mondialisation des échanges.

\section{Relation de service et interdépendances}

Néanmoins, ces différents travaux proposent une explication principalement «fonctionnaliste » du jeu concurrentiel, où les configurations d'échange répondent aux éléments de contexte économique ou à la nature des activités. Nous avons souhaité contribuer à ce débat en mettant en valeur les processus par lesquels s'opèrent l'articulation entre coopération et concurrence ainsi que les rééquilibrages entre ces deux dimensions. Plus précisément, nous nous intéressons aux stratégies des clients et des fournisseurs pour agir sur les interdépendances au sein d'une relation de coopération.

Notre analyse des interdépendances s'inspire des investigations qualitatives des échanges dans les relations entre partenaires (Uzzi 1997, Gadde et ali, 2003). Elle part du constat que, dans une relation interindustrielle, la division formelle des tâches entre fournisseur et client est incomplète et laisse dans l'opacité un enchevêtrement d'interactions entre les deux organisations. La relation se caractérise par un investissement du fournisseur dans une «relation de service» (De Bandt, Gadrey, 1997) visant à prendre en charge les nombreux ajustements qui facilitent la coordination des activités.

Deux catégories empruntées à Hatchuel (1998) permettent de caractériser les relations d'échange : on parlera de «coprescription» quand le client et le fournisseur définissent ensemble le contenu de la prestation, articulent leurs compétences techniques pour qualifier les besoins et définir les solutions par exemple dans le co-développement de produits (Calvi, 2000) ou d'équipements industriels, et «coproduction» quand il existe des activités opérationnelles partagées, que les systèmes sociotechniques des deux entreprises sont fortement inter-reliés, qu'il y a des ajustements informels sur les flux et sur la qualité.

La coprescription et la coproduction introduisent des interdépendances entre partenaires. Ainsi, la «coprescription » est particulièrement importante quand le client a un besoin spécifique - et donc ne peut se saisir d'une offre générique proposée par le marché - et qu'il n'a pas les compétences suffisantes pour définir une solution technique. Un unique aller-retour entre le 
client et le fournisseur (où le client interne élaborerait le cahier des charges, le fournisseur une unique réponse) ne suffit pas à explorer complètement la spécificité des besoins et à préciser l'offre. Le fournisseur investit des compétences et du temps pour aider le client à définir ce qui fera l'objet de l'échange ${ }^{2}$. Ce travail comprend généralement les deux termes de la «singularisation» (Karpik, 2007): il y a un effort de «personnalisation » particulièrement coûteux, qui s'accompagne en général de la mise en valeur d'une certaine «originalité » des compétences du fournisseur.

La « coproduction » rassemble tous les ajustements nécessaires pour gérer les aléas imprévus de la relation. Au sein de l'organisation cliente, les destinataires du produit ou de la prestation (par exemple, les activités de production) y sont en général très sensibles : ils savent que la qualité des composants achetés est déterminante pour leur activité, mais que celle-ci n'est jamais fixée une fois pour toute. Ils souhaitent aussi bénéficier d'améliorations proposées par les fournisseurs. La «coproduction» est très sensible dans les activités de maintenance où les équipes du sous-traitant interviennent en coordination avec les équipes de production du client. Ainsi la «coproduction » porte-t-elle sur la part non spécifiée par le contrat.

Les besoins des clients de coproduction et de coprescription sont autant de portes ouvertes dans lesquelles les fournisseurs s'engouffrent pour développer les relations avec leur client et accroître la dépendance de ces derniers (Neuville, 1998). Certains prestataires interrogés utilisent d'ailleurs le terme d'«intimité client» pour exprimer cette stratégie commerciale, reprenant les termes du marketing d'affaires (Azimont et al. 1999) : le «maillage» de l'organisation client permet d'identifier tous les acteurs significatifs dans les décisions ou d'influencer le besoin du client. Cette «intimité » est d'autant plus forte que le fournisseur est présent chez le client : les acteurs opérationnels qui interviennent dans les prestations (chef de projet, chef de chantier, responsables d'équipe de maintenance) sont déjà en lien avec des correspondants clients avec qui ils partagent bon nombre d'activités au jour le jour.

Néanmoins, l'intensité de la coprescription et de la coproduction peut évoluer : client et fournisseur peuvent agir sur la répartition des tâches, leur formalisation. Les relations

\footnotetext{
${ }^{2}$ Ces pratiques de coprescription se développent de façon significative dans la mesure où les donneurs d'ordre ont de moins en moins de compétences techniques sur ce qu'ils achètent, à tel point qu'ils ne sont plus capables, seuls, de spécifier leurs besoins.
} 
d'interdépendance ne sont pas immuables. L'objet de l'échange est malléable, autant que la portée des engagements ${ }^{3}$.

\section{Cadrage et désingularisation}

Pour réintroduire la concurrence et disposer d'alternatives attractives, l'acheteur peut agir sur les échanges informels, sur le caractère spécifique de l'échange ou sur la configuration des échanges.

Comme nous l'avons montré plus haut, la «relation de service» favorise une prolifération d'échanges informels et d'agencements sociotechniques (Comet, 2007) qui permettent l'articulation entre le système de production de l'entreprise cliente et le système de production des fournisseurs. Le travail de l'acheteur peut répondre à cette dynamique par des actions de «cadrage » (Callon, 1999) qui réintègrent dans l'interaction marchande la prolifération des échanges «externes » à celle-ci (les « externalités»). Cela peut se traduire sous la forme d'une rationalisation de ces échanges informels afin de les intégrer dans le cahier des charges ou les critères de sélection des fournisseurs ${ }^{4}$.

L'acheteur peut agir sur le processus de singularisation (Karpik, 2007) par des actions de « désingularisation » des échanges pour accroître l'interchangeabilité (et la possibilité de mettre en concurrence). Cela peut se décliner de la façon suivante: l'acheteur tente de réduire la singularité de l'expression des besoins et des propositions de solution des fournisseurs. Il cherche à rendre comparables des offres spécifiques, à dénouer des agencements sociotechniques trop spécifiques qui contribuent à la « capture » (Trompette, 2005).

Enfin, l'intervention de l'acheteur concerne aussi l'organisation de la configuration des échanges. Une fois que les relations sont réaménagées pour réduire les interdépendances informelles existantes, l'acheteur sollicite les nouveaux fournisseurs, en leur facilitant l'accès à son entreprise, en soutenant le travail de « singularisation » de leur offre...

\footnotetext{
${ }^{3}$ La « relation de service » se différencie des partenariats ou des alliances en recherche et développement qui font l'objet d'une reconnaissance formelle (et qui impliquent par exemple des dépôts de brevets communs).

${ }^{4}$ Les opérations que nous observons tiennent un rôle similaire aux activités de cadrage observées sur les marchés des produits de consommation, activités qui dénouent les liens entre les personnes, entre les personnes et les choses et permettent la circulation des biens et des services, la comparaison des alternatives et la formation du prix (Weber, 2000, Garcia 1986).
} 


\section{Acheteurs et clients internes}

Chez le client, l'acheteur n'est pas le seul acteur concerné par la relation avec les fournisseurs. Nous proposons donc d'entrer dans l'organisation interne du client et distinguer plusieurs logiques d'action pour comprendre le «comportement» (Cyert, March. 1963) du client. Johnston et Bonoma (1981) et Dubuisson-Quellier (1999) soulignent la diversité des rôles possibles dans le processus de décision de choix d'un fournisseur. Il n'est pas sûr que l'acheteur soit l'acteur le plus puissant. Ainsi Pettigrew (1975) montre que l'acteur impliqué dans la négociation technique et porte-parole du fournisseur auprès de sa direction, le «technical gatekeeper », joue donc un rôle déterminant dans la décision d'achat (même dans le cas d'un contrat de prestation tout à fait stratégique pour l'entreprise).

Nous proposons de regrouper dans une catégorie générique «clients internes", les «prescripteurs », qui valident les solutions proposées et les «bénéficiaires », qui utilisent concrètement dans leur activité la prestation ou le produit. Dès qu'il s'agit de modifier le contenu des échanges, la frontière entre les activités du fournisseur et du client, les clients internes sont directement concernés.

Aussi, nous pouvons faire l'hypothèse que la contradiction entre les logiques de coopération et de compétition se reporte sous la forme d'une tension entre «clients internes » et acheteurs : les «clients internes» sont généralement inscrits dans des relations d'échanges avec les fournisseurs en place, ils sont les principaux bénéficiaires de la relation de service, tandis que les acheteurs, en partie affranchis de ces relations de dépendance, tentent d'ouvrir le jeu à de nouveaux fournisseurs.

Les activités de l'acheteur ont donc une portée stratégique : elles concourent à dénouer les relations d'échanges stratégiques entre fournisseurs en place et clients internes et nouer de nouvelles relations avec des fournisseurs alternatifs.

\section{Méthode de recherche : analyser les interdépendances entre acheteurs, clients internes et fournisseurs, et leurs transformations}

Nous présentons ici notre méthode de recherche fondée sur la construction et la comparaison de cas : réalisation des entretiens, sélection des cas, formalisation et comparaison. 


\section{Un échantillonnage raisonné de cas}

Notre démarche d'enquête s'appuie essentiellement sur des entretiens approfondis réalisés auprès d'acheteurs, de commerciaux et de responsables techniques, à propos de situations singulières de choix de fournisseurs, de changements ou de renégociation ${ }^{5}$.

Le choix des cas s'inspire de la méthode d'échantillonnage raisonné proposée par Strauss et Corbin (1990). Au fur et à mesure de la recherche, alors que nous collections les données, et que nous élaborions des interprétations, nous avons focalisé notre intérêt sur les cas qui pouvaient éclairer ou introduire de la variété pour telle ou telle catégorie d'analyse.

Notre objectif étant d'analyser comment les acheteurs interviennent pour «organiser le marché », nous avons recherché des situations «réversibles», où les fournisseurs étaient impliqués dans une «relation de service », mais où il n'y avait pas de dépendance économique excessive des clients en termes de part de marché, de taille d'entreprise, de spécialisation technique ${ }^{6}$. Pour l'ensemble des cas étudiés, il existe, du point de vue de l'acheteur, plusieurs fournisseurs alternatifs ayant des compétences comparables. Les fournisseurs interrogés dans chacun des cas identifient aussi l'entreprise comme un client potentiel, qui fait partie de leur stratégie de marché, soit parce qu'elle est déjà en place, soit parce qu'elle cherche à développer son activité.

Nous recherchions des situations où les rôles des acheteurs étaient bien différenciés des « clients internes ». La tension entre engagement et compétition est d'autant plus visible que les acteurs internes, l'acheteur d'un côté, les «clients internes » de l'autre, ne défendent pas la même logique, ne développent pas les mêmes types de liens avec les fournisseurs ${ }^{7}$. L'échantillon des cas a aussi été constitué de façon à refléter des pratiques contractuelles différentes, et pour

\footnotetext{
${ }^{5}$ Certains entretiens ont été réalisés à l'occasion d'une recherche pour le compte d'une entreprise de service, qui cherchait à comprendre les pratiques commerciales de ses filiales et à évaluer la satisfaction de ses clients. Les autres entretiens ont été réalisés grâce à des contacts obtenus auprès d'acheteurs en formation continue.

${ }^{6}$ Le seul cas particulier est le cas Mecano, sous-traitant de maintenance, qui a un nombre limité de clients. Le client étudié est celui qui a la part de marché la plus importante. Le sous-traitant est donc fortement dépendant économiquement de son client.

${ }^{7}$ Nous avons mis de côté les situations où par exemple, l'acheteur a une compétence technique importante et est en mesure de définir complètement les besoins sans consulter en interne, ou bien les situations où l'acheteur est totalement absent et laisse au client interne (un service technique, une ligne de production) un contrôle total de la relation du début jusqu'à la fin.
} 
chacune d'entre elles, disposer de plusieurs cas contrastés. Par exemple nous souhaitions comparer des situations où le processus de mise en concurrence avait été mené à son terme et des processus où il avait été abandonné ${ }^{8}$.

\section{Décrire le système acheteur - client interne - fournisseur en place - fournisseur alternatifs}

Pour chacun des cas, nous avons cherché à réaliser des entretiens semi-directifs avec l'acheteur, le client interne et un des fournisseurs (fournisseur en place ou fournisseur alternatif). Nous avons pu interroger l'acheteur et le client interne dans tous les cas. Nous n'en avons pas toujours pu rencontrer les fournisseurs. Dans trois cas (Color, Electro et Waste) l'acheteur ne nous y a pas autorisé ${ }^{9}$. Dans les autres cas, il s'agissait du fournisseur qui a remporté le marché (Maintini, Informa, Renovbati). Pour Renovutil, les commerciaux interrogés ont connu deux situations : l'une où il a gagné le marché (en position d'outsider) et l'autre où il l'a perdu (toujours en position d'outsider).

Par ailleurs, nous avons accumulé une vingtaine d'autres entretiens approfondis auprès d'acheteurs, de clients internes ou de fournisseurs, toujours à propos de choix ou de changement de fournisseur, mais sans qu'il nous soit possible d'interviewer les autres acteurs impliqués. Nous avons essentiellement retenu de ces entretiens des éléments sur les pratiques des acheteurs, mais aussi des récits permettant de conforter notre analyse des cas.

Généralement, les entretiens se focalisent sur un récit du choix du fournisseur et de la mise en place de la prestation: nous questionnons les acteurs sur les différentes alternatives, sur le déroulement du changement, les difficultés techniques et contractuelles à résoudre, les résistances rencontrées, les conflits internes, les conflits entre entreprises... Les récits des différentes parties prenantes ont été confrontés les uns aux autres, afin de reconstituer plus fidèlement les événements mais surtout de dégager les objectifs propres à chacun en situation.

\footnotetext{
${ }^{8}$ Voir le tableau qui présente les cas et les entretiens réalisés en annexe. Par souci de clarté et de simplicité, nous désignons dans cet article chaque cas par un nom fictif accordé au fournisseur, en lien avec son activité. Par souci de simplicité, nous ne nommons pas les entreprises clientes (qui sont néanmoins toutes différentes).

${ }^{9}$ Accéder à des processus de sélection de fournisseur n'est pas facile. Il existe une large part d'opacité des acteurs les uns vis-à-vis des autres même en interne. Pour construire mon enquête j'étais dépendant de mes « relais ». Dans quelques cas, mes relais étaient les acheteurs, dans d'autres cas un fournisseur. Mon objectif principal était de pouvoir réaliser des entretiens approfondis avec l'acheteur et le client interne et d'avoir des points de vue contrastés sur le processus de changement de fournisseurs. Les entretiens avec les fournisseurs n'ont pas toujours été possibles suivant le mode d'accès au terrain.
} 
Les entretiens ont été menés rétrospectivement après le démarrage de la prestation du fournisseur.

Lors des entretiens, nous avons aussi demandé aux acheteurs de nous raconter leur activité quotidienne, d'expliciter leurs instruments et le contexte organisationnel de leur action. Nous nous sommes concentrés sur les activités qui organisent la mise en concurrence des fournisseurs ${ }^{10}$.

Les entretiens confirment rapidement que les principaux acteurs impliqués chez le client sont les bénéficiaires directs de la prestation ou de l'achat et les services techniques qui définissent les besoins. Le rôle des dirigeants est évoqué dans certains cas particuliers. Ils interviennent de deux façons : définir la mission de l'acheteur, intervenir en arbitre en cas de conflit entre acheteurs et clients internes. Néanmoins, ils n'interviennent pas directement dans la relation : dans les cas étudiés, il ne s'agit pas de prestations suffisamment stratégiques pour que les dirigeants interviennent directement ou mobilisent leur propre réseau.

Pour chaque cas, nous avons réalisé une cartographie du système d'action, avec les acteurs impliqués, leurs enjeux et leurs relations de dépendance, leurs stratégies. Cette cartographie est indicative car les relations évoluent dans le temps. Les sociogrammes proposés en annexe tentent de représenter les situations de transition (cas Renovutil et Color) : ils nous ont permis de saisir comment les relations évolues au fur et à mesure du processus de mise en concurrence.

C'est pourquoi nous avons formalisé les cas sous la forme de récits, mettant en valeur les efforts de rationalisation, les investissements des acteurs dans de nouvelles relations, les conflits et les stratégies de blocage, les négociations et prises de décision. Cette méthodologie suit les propositions de Langley (1999) concernant l'usage des analyses de processus pour des approches qui relèvent de la «grounded theory ». Ces récits nous ont permis de dégager des régularités dans les actions engagées, les jeux d'acteurs, les situations qui en résultent. Ces récits ont permis un travail de comparaison (Eisenhardt, Graebner 2007) et l'explicitation au fur et à mesure quelques propositions sur les conditions qui facilitent ou entravent l'organisation de la compétition entre fournisseurs. Le tableau 2. en annexe reprend l'ensemble des cas et les principales catégories qui nous permettent de les comparer.

\footnotetext{
${ }^{10}$ Nous avons donc mis de côté la part administrative du travail de l'acheteur, qui comprend le formalisme du contrat et de la commande, le suivi de l'exécution des contrats, ainsi que l'organisation du paiement.
} 


\section{La mission de la fonction Achats : organiser la mise en concurrence}

Les acheteurs ne se définissent plus seulement comme des négociateurs de contrats. Ils défendent un nouveau rôle, plus large, plus stratégique. Ils ont pour mission de replacer les relations avec les fournisseurs dans le cadre de marchés globalisés et d'organiser le mieux possible la compétition entre fournisseurs.

\section{Les «politiques d'achats » visent la globalisation}

Dans les cas étudiés, les «politiques d'achats » visent l'organisation de marchés amont plus «globalisés » par une centralisation des décisions achats à un niveau au moins européen. La centralisation de la fonction Achats consiste à faire passer l'activité d'achat du niveau des sites industriels à un niveau centralisé. Elle permet d'élargir le marché pertinent sur lequel l'entreprise s'approvisionne. En se centralisant, la fonction Achats ne se retrouve plus face à un ensemble de quasi-monopoles locaux ou nationaux mais face à un marché potentiellement beaucoup plus concurrentiel. Dans ce contexte, les politiques internes de réduction du nombre de fournisseurs réactivent le jeu concurrentiel. L'acheteur européen a aussi la possibilité de faire évoluer la part de marché de chaque fournisseur et bénéficie donc d'un bon levier d'incitation, tout en n'ayant pas besoin de rechercher de nouveaux fournisseurs ${ }^{11}$.

Certains acheteurs rencontrés travaillent également dans le cadre d'une politique d'approvisionnement dans les pays dits «à bas coûts de main d'œuvre ». Cette politique vise à tirer profit des écarts de coûts, essentiellement de main d'œuvre. Désormais, la taille du marché pertinent est internationale.

\section{La fonction Achats doit rendre des comptes sur sa capacité à maintenir un haut niveau de compétition entre fournisseurs}

Les acheteurs sont soumis à une multitude d'indicateurs de performance. Traditionnellement, un des indicateurs de performance, la réduction des coûts, joue le rôle «d'indicateur prégnant » (Boussard, 2001) : non seulement il contribue au contrôle de leur activité, mais il fait partie de l'identité professionnelle des acheteurs. Plus précisément, les acheteurs sont conduits, la plupart du temps, à raisonner en «coût d'achat». Certains indicateurs de suivi de leur activité (les « saving ») les conduisent à se concentrer sur le prix et à favoriser les offres les moins chères.

\footnotetext{
${ }^{11}$ Leur travail est facilité par des stratégies de conquête de part de marché, chez les fournisseurs, en particulier dans le contexte d'expansion des activités à l'international.
} 
Certes, cet indicateur fait aussi l'objet de stratégies de contournement, comme celle décrite ${ }^{12}$ par M. Berry (1983), mais il reste central dans la définition de la fonction, d'autant plus que le développement massif des systèmes d'information, la centralisation des achats et le travail de segmentation facilitent l'accumulation d'informations sur les prix, le calcul et le « reporting» des gains.

Les objectifs fixés aux acheteurs évoluent : les directions des achats n'hésitent pas à introduire de nouveaux indicateurs qui visent non pas les résultats de réduction des coûts (dont la mesure reste problématique) mais la pratique concurrentielle elle-même, en définissant un taux de renouvellement des fournisseurs, ou le taux de fournisseurs de pays dits « low cost». Le travail de l'acheteur est mesuré en fonction de son activité d'organisation et de mise en concurrence, activité considérée comme beaucoup plus exigeante et plus efficace que la renégociation des prix avec les fournisseurs existants.

Pour autant, de nombreuses directions Achats ont compris qu'elles ne parviendront à développer leur rôle qu'à condition de s'intéresser davantage aux dimensions hors-prix dans la comparaison des offres ${ }^{13}$. La prise en compte des dimensions hors prix est encouragée par le fait que les acheteurs sont souvent sensibles à la menace d'un «rejet» du nouveau fournisseur par les clients interne (ces derniers ont souvent la possibilité d'exploiter les failles du nouveau fournisseur, de le mettre en échec, ce qui aura pour effet de désavouer le travail de l'acheteur).

\section{Le travail de segmentation met en forme les marchés amont}

En amont de la négociation avec les fournisseurs et avec les clients internes, les acheteurs tentent de se donner une représentation des marchés. Tout manuel décrivant le métier d'acheteur commence par la présentation de l'activité de segmentation des achats, base de l'organisation et de la stratégie d'achat. Il ne s'agit pas d'identifier des marchés au sens strict du terme, mais de regrouper des fournisseurs et des produits selon leur caractère potentiellement substituables.

Sur chaque segment, l'acheteur raisonne comme s'il était face à un marché en étudiant sur ce marché sa «position concurrentielle» en tant que client : quels sont les fournisseurs présents,

\footnotetext{
${ }^{12}$ Une stratégie de manipulation de l'indicateur consiste à définir avec un fournisseur des prix élevés pour les nouveaux produits (avec une marge élevée pour le fournisseur) pour obtenir de ce même fournisseur des réductions de prix sur les produits existants (cela permet à l'acheteur de tenir ses objectifs de réduction de coût, calculés sur les produits achetés d'une année sur l'autre, tout en laissant au fournisseur une marge globale constate).

${ }^{13}$ Cette préoccupation est d'ailleurs relayée dans les manuels professionnels, dans les formations des acheteurs et par les consultants qui pratiquent les audits internes de cette fonction.
} 
quel est leur chiffre d'affaire, quelle est sa part de marché en tant que client avec chacun d'entre eux, quels sont les prix, quelle est leur stratégie de développement. Il s'intéresse à leur solidité financière, à la fiabilité de leurs approvisionnements. Il essaie aussi d'évaluer leur degré de spécialisation technique. Ce travail est facilité quand l'acheteur appartient à un groupe industriel implanté dans plusieurs pays : il s'agit essentiellement de centraliser l'information issue des correspondants locaux.

Dans le cas Renovutil, l'acheteur France tente d'identifier l'ensemble des acteurs spécialisés dans le champ des utilités industrielles sur le marché français. Il existe plusieurs grands groupes et des prestataires locaux. Deux prestataires sont déjà présents dans plusieurs usines du groupe, l'un est déjà implanté dans la région, l'autre a une forte stratégie de croissance. Il consultera en priorité ces deux prestataires. Il ne néglige pas pour autant les quelques prestataires locaux. Son objectif est d'obtenir au moins 3 offres de qualité pour un projet de modernisation.

Ainsi le premier volet de l'activité de l'acheteur vise à configurer des espaces de marché où la concurrence peut être organisée. Elle place côte-à-côte des fournisseurs comparables. Elle permet de constituer des ensembles de fournisseurs entre lesquels le jeu concurrentiel peut avoir un sens. Elle ne cherche pas non plus à établir une représentation complète des alternatives, mais une représentation «satisfaisante»: un acheteur se limite généralement à 3 ou 4 fournisseurs alternatifs, étant entendus que ces fournisseurs doivent aussi répondre aux critères de la politique Achats (par exemple en termes de choix de pays, de réduction du nombre de fournisseurs...).

Ces premières opérations de «cadrage » produisent des représentations des marchés potentiels. Ce n'est pas parce que deux fournisseurs appartiennent au même segment, sont considérés comme «proches », qu'ils répondront de la même façon aux besoins de la production. Ce n'est pas parce qu'un fournisseur répond de façon satisfaisante à une usine que ce sera le cas pour une autre usine dans une autre région. Reste à concrétiser ces marchés par des actions de transformation afin d'accroître l'interchangeabilité entre les fournisseurs.

\section{«Dénouer » les relations existantes : la rationalisation et la standardisation du contenu des échanges}

Quand les organisations des clients et des fournisseurs sont enchevêtrées, que le fournisseur en place participe par de nombreux ajustements informels aux objectifs de ses interlocuteurs, réintroduire de la concurrence n'est pas aisé. L'acheteur va donc s'attaquer à ces relations, tenter de les réorganiser, les réduire, les simplifier... afin d'obtenir une plus grande modularité et une plus grande interchangeabilité. 


\section{L'effort de rationalisation interne simplifie les interfaces avec les fournisseurs}

Rendre possible l'interchangeabilité des fournisseurs suppose d'abord d'agir sur l'organisation interne afin de faciliter l'intégration de nouveaux fournisseurs. Un premier travail consiste donc à formaliser les activités aux interfaces. Ce qui comprenait hier une part d'ajustement informel, est progressivement rationalisé, simplifié, de telle façon qu'il soit possible de spécifier à un nouveau fournisseur les activités attendues ou de comparer deux fournisseurs en place.

Dans les cas étudiés, la situation de «coproduction » la plus flagrante est la sous-traitance de la maintenance (Mecano et Maintini) : la répartition des tâches n'est pas toujours stabilisée, les interventions de maintenance préventive dépendent de la disponibilité des machines ; la production est dépendante de la réactivité de la maintenance pour les opérations curatives, enfin la durée de vie des machines dépend à la fois des réglages par la production et de la compétence de la maintenance. Dans les deux cas étudiés, avant d'organiser un appel d'offre pour mettre en concurrence de nouveaux fournisseurs, et contractualiser avec eux sur la base d'un contrat au forfait, il était envisagé de planifier l'activité de maintenance, de préciser les volumes de main d'œuvre impliquée et donc de dimensionner le «contrat au forfait». Dans le cas de Mecano, ce travail de rationalisation a échoué, compte tenu de la complexité des imbrications entre le planning de la production et celui de la maintenance. Dans le cas de Maintini, au contraire, c'est le fournisseur alternatif qui, ayant évalué les risques d'un contrat au forfait mal défini, a obtenu de travailler d'abord en régie et a entrepris, avec sa propre compétence et ses outils de gestion, le travail de formalisation des besoins du client.

Dans le cadre des politiques de centralisation des achats et de réduction du nombre de fournisseurs, cette rationalisation s'accompagne d'une standardisation des termes de l'échange, ce qui peut avoir des implications importantes en interne puisque les activités s'organisent souvent autour des offres de service des fournisseurs. Plus l'activité est «coproduite», plus le travail de rationalisation est fastidieux. La standardisation suppose dans certains cas de réintégrer des activités auparavant déléguées aux fournisseurs, ou à en déléguer de nouvelles.

Cette action de rationalisation et de standardisation est facilitée quand les fournisseurs alternatifs sont déjà présents dans l'entreprise. L'acheteur peut assez facilement comparer les fournisseurs présents, évaluer la qualité de leurs services, et déterminer le niveau de qualité attendu. Il lui est plus facile de démontrer, par exemple, qu'un site industriel peut se passer d'un service de haute qualité proposé par un fournisseur particulièrement cher, s'il existe un autre site industriel, dans la même entreprise, où le client interne ne bénéficie pas de ce service.

Le cas Color illustre particulièrement ce phénomène de standardisation des besoins internes. Le fournisseur en place était parvenu à un haut degré de qualité de rendu avec ses peintures sur les pièces des clients (pièces plastiques destinées à l'industrie automobile), il connaissait parfaitement les lignes de peinture de son client et avait adapté la formulation de ces peintures à ces lignes, aux 
matériaux peints, aux rendus attendus. Quand la Direction des Achats de l'entreprise a imposé à l'usine cliente de laisser Color pour un autre fournisseur, elle a provoqué un vif conflit avec l'usine. Les premiers essais avec les peintures standards proposées par le nouveau fournisseur de peinture conduisaient d'ailleurs à des rendus des peintures particulièrement médiocres. L'usine souhaitait rester avec le fournisseur actuel dont elle connaissait les compétences et le travail de formulation spécifique à ses besoins. Cependant, la Direction des Achats, engagée avec le nouveau fournisseur, n'est pas revenue en arrière. Elle a estimé que l'usine devait s'adapter, le nouveau fournisseur ayant fait ses preuves avec une autre usine au sein du même groupe. Un acheteur a été délégué sur place pour organiser dans l'urgence un travail de fiabilisation de ces nouvelles peintures avec le laboratoire de l'usine. Des essais de formulation ont été systématisés. Il y a eu un apprentissage interne significatif et cet apprentissage a permis d'obtenir, avec les produits standards, une qualité identique à ce que l'usine obtenait avec le fournisseur précédent.

Dans les cas analysés, le travail de rationalisation connait, selon le niveau d'interdépendance entre les activités, des succès divers. Cependant, une rationalisation limitée n'empêche pas les acheteurs d'engager un processus de changement de fournisseur. De nombreux ajustements ont lieu à l'issue du basculement, avec une dégradation (en général momentanée) de la performance.

C'est pourquoi les politiques de standardisation sont «pragmatiques » : elles expérimentent la possibilité de standardiser les produits, les contrats, d'évoluer vers des contrats globaux (par exemple, au niveau européen), elles vérifient les gains d'échelle et de pouvoir de négociation, elles évaluent le coût de la centralisation et de la standardisation, avant de passer à une logique systématique $^{14}$.

\section{L'objectivation de l'évaluation des fournisseurs facilite l'interchangeabilité}

La rationalisation des organisations internes et la simplification des besoins ont des limites : il n'est pas possible de spécifier toutes les articulations entre activités de production. Il reste toujours des ajustements informels. Il convient de s'assurer que les fournisseurs alternatifs soient capables de répondre aux attentes (explicites ou non) en termes de «coproduction». Il s'agit de sélectionner ceux qui sont capables d'offrir le niveau de service attendu ou d'accompagner le fournisseur outsider de façon à ce qu'il assure ce niveau. Cette démarche

\footnotetext{
${ }^{14}$ Dans le cas Color, le processus de standardisation est allé jusqu'à une inversion de la prescription, au sens où c'est le fournisseur qui impose son standard. On retrouve ce phénomène dans d'autres secteurs, comme l'électronique grand public, où le processus de modularisation des produits et de standardisation des composants a permis la constitution de marchés concurrentiels mondiaux (Berger 2005).
} 
prolonge le travail de normalisation (Segrestin, 1997) qui avait déjà permis une certaine ouverture des relations entre partenaires, mais elle va aujourd'hui beaucoup plus en profondeur. Plus la dimension coproduite est importante, plus la mise en compétition porte sur les fournisseurs et non les offres : il ne s'agit pas de choisir une offre mais une entreprise, en fonction de ses compétences, de sa notoriété. Dans la mesure où l'acheteur assume le fait que l'activité est en grande partie « coproduite », il invite généralement les principaux intéressés, les clients internes, à évaluer les fournisseurs.

A ce niveau, l'acheteur cherchera cependant à montrer que plusieurs fournisseurs auront les capacités et joueront le jeu de la « coproduction ». Ces fournisseurs ont le droit de concourir, ils ont été sélectionnés dans le cadre d'une première compétition entre organisation, le «marché d'élection» (Mariotti, 2005). Le but de l'acheteur est d'obtenir l'accord des acteurs internes (clients internes, management) sur les critères d' «élection », puis sur la liste d' "élus », afin d'organiser plus facilement ensuite, entre eux, la compétition sur les offres techniques et les offres de prix, et leur «allouer» les marchés en fonction des critères de prix. C'est à cette condition que l'acheteur peut reprendre la main.

Les fournisseurs peuvent aussi contribuer à l'objectivation de cette «coproduction » en termes de critères de performances ou de critères de satisfaction, à destination des clients internes et des acheteurs, à condition bien sûr que de tels critères les différencient. Mais ce faisant, ils facilitent le travail d'objectivation des critères, de comparaison et donc de mise en compétition.

Maintini gère ses interventions de maintenance avec un logiciel spécialisé qui trace la nature des interventions en maintenance curative, les délais d'intervention, mais aussi des indicateurs globaux de temps de disponibilité des machines, qui reflètent aussi ses actions en termes de maintenance préventive. Avec certains de ses clients, Maintini s'est engagé à améliorer le niveau de performance des procédés (consommation énergétique...) avec comme hypothèse que ses compétences de maintenance et de réglage contribuent à une part essentielle de cette performance. Le contrat comporte une prime indexée sur ces critères de performance. Ainsi, en définissant ses critères de performance, Maintini est en mesure de faire reconnaître pleinement à son client sa contribution à la performance.

La formalisation des caractéristiques hors-prix, si elle permet une discussion interne, n'est jamais poursuivie jusqu'au bout, par exemple par une « monétarisation » des forces et faiblesses de chaque fournisseur, une sorte de «coût complet» permettant une parfaite mise en 
équivalence d'offres hétérogènes en termes de qualité et de prix. ${ }^{15}$ Les acteurs internes se contentent de critères hétérogènes dont le prix fait partie.

\section{La rationalisation s'étend aux fournisseurs outsiders pour faciliter leur intégration}

Le travail sur les fournisseurs ne se limite pas à la prospection et la sélection. L'effort peut aussi porter sur l'organisation et les capacités des fournisseurs alternatifs, il s'agit de les accompagner pour qu'ils atteignent rapidement le niveau d'exigence de l'entreprise. Le travail sur l'organisation $\mathrm{du}$ fournisseur est particulièrement important quand il s'agit des politiques d'achat à « low cost». Ces politiques s'appuient en général sur des programmes qui visent à mettre ces nouveaux fournisseurs au niveau des fournisseurs en place en termes de logistique et de qualité de produits, en s'appuyant sur des normes de management mais aussi sur des compétences techniques des clients. C'est le rôle de la fonction «qualité achat (dépendante hiérarchiquement de la fonction Achats), qui intervient chez le fournisseur comme « consultant» et prend en charge ce travail de «qualification », qui permet de faire entrer un nouveau fournisseur. De plus en plus fréquemment, en particulier dans la filière automobile et dans les prestations en informatique, le travail de mise à niveau des fournisseurs «low cost» s'appuie sur des partenaires existants, encouragés à développer un certain pourcentage de leurs activités dans ces pays, soit par acquisition d'entreprises locales, soit en développant une nouvelle activité.

Ainsi, la standardisation et la formalisation des critères d'évaluation et des processus transversaux aux deux organisations réduisent la prolifération des «débordements » - ici, les ajustements informels - afin de rendre les fournisseurs substituables. Elles permettent aussi de réduire, de simplifier et d'objectiver les caractéristiques hors-prix qui seront prises en compte dans la comparaison et le choix des fournisseurs, et donc de réduire la singularité de chaque relation ${ }^{16}$.

\footnotetext{
${ }^{15}$ Le travail nécessaire pour apprécier en termes économiques des différences qualitatives entre fournisseurs est trop important pour être réalisable. Il supposerait l'élaboration de différents scénarios d'organisation de l'activité (activités prises en charge en interne ou par le fournisseur, synergies possibles...) et le rapprochement de ces scénarios avec des données comptables internes, pour valoriser les différentes options.

${ }^{16}$ La rationalisation tant interne qu'externe de la relation est facilitée par une certaine stabilisation des exigences de gestion logistique et de qualité, et donc la maturité technique et organisationnelle du secteur.
} 


\section{La mobilisation des fournisseurs dans une « coprescription ouverte »}

La notion de «coprescription»s'intéresse aux situations où le fournisseur contribue à la définition des besoins du client et à la formalisation des engagements contractuels. Les relations de coprescription sont en général favorables aux fournisseurs en place: ils connaissent l'organisation, les équipements, et n'ont pas besoin d'investir autant de temps pour construire leur offre. Grâce aux relations interpersonnelles nouées avec les clients internes, ils cherchent à obtenir un engagement informel de leur part.

« Je suis déjà au courant avant même qu'il y ait l'idée de démarrer le projet j'ai des infos dessus. Les gars me tiennent au courant, c'est le deal qu'on a ensemble. Jean m'appelle pour me dire que potentiellement des projets vont démarrer, ça me permet d'anticiper, de voir les consultants, on essaye de mieux comprendre techniquement, on rentre en contact avec les techniciens pour obtenir des infos. Une fois qu'on l'a bien compris on voit comment on va s'organiser, quels sont les lots. » (Commercial, cas Informa)

Comment l'acheteur peut il gérer cette relation précontractuelle entre clients internes et fournisseurs en place et éviter qu'elle n'entraine une dépendance vis-à-vis d'une offre singulière?

\section{L'organisation d'une coprescription ouverte}

Les acheteurs tentent d'organiser ce que nous appellerons une « coprescription ouverte »: mobiliser plusieurs fournisseurs en parallèle dans la coprescription, plutôt que de rester prisonnier d'un seul interlocuteur. L'acheteur essaie de reprendre le cahier des charges avec le client interne pour le formuler en des termes plus génériques, plus standardisés, qui précisent davantage les fonctionnalités attendues que les solutions techniques pour les atteindre ${ }^{17}$, acceptant une plus grande diversité de répondants que le cahier des charges initial. Il s'agit d'impliquer plusieurs fournisseurs (en général 2 ou 3) dans un dialogue approfondi pour enrichir et élargir le cahier des charges, mais aussi recueillir des propositions techniques de solutions.

Les acheteurs ont besoin de l'aide des fournisseurs alternatifs pour s'affranchir des clients internes et des fournisseurs en place. C'est grâce aux idées qu'ils proposent que l'amélioration du cahier des charges est possible. La «coprescription ouverte » réduit le risque d'un besoin

\footnotetext{
${ }^{17}$ Ainsi, le cahier des charges « fonctionnel » se différencie du cahier des charges «techniques », dans la mesure où le premier essaie de caractériser les fonctions attendues de l'objet (résistance, comportement...) et non des caractéristiques techniques afin de laisser aux fournisseurs le soin de proposer différentes solutions techniques, comme le choix des matériaux, le dimensionnement...
} 
mal spécifié, soit parce qu'il serait trop précis (il réduirait le nombre de fournisseurs à un seul) soit parce qu'il ne serait pas assez précis (il ne permettrait pas d'établir les engagements contractuels).

Un chef de projet raconte le processus de consultation par un client pour un projet d'externalisation et de modernisation des utilités industrielles: «Au départ, les seuls besoins exprimés étaient l'objectif de réduction de coût. A ce stade, on ne connaît pas l'usine, on ne sait pas comment ils sont organisés et ce que l'on peut réellement faire (...) On a essayé de créer de l'intimité avec le client : voir si réellement il y a un projet, voir et comprendre ce qu'ils veulent. Ensuite, 3, 4 mois plus tard, on a été chez eux, on a fait des études, des audits techniques, des analyses avec les ingénieurs d'exploitation, des experts en charge d'autocontrôle (...). Ensuite, après la présentation de nos premiers résultats, le client a décidé de lancer un appel d'offres. Ils voulaient aussi nous tester et voir ce que les autres pouvaient apporter. Nous avons tout fait pour leur dire que c'était une bêtise de lancer un appel d'offres. Ca n'a pas marché, nous avons perdu cette bataille ! Mais, c'est courant ; dans le monde industriel (...). Première phase : 4 entreprises sélectionnées. Nouvelle phase : plus que 2 entreprises sont en compétition. Entre le premier et le second tour, beaucoup de travail : des éclaircissements sur notre offre, retravailler sur de nouvelles pistes, écrire des contrats, faire un business plan financier, revalider les économies »

Lors d'investissements importants, et à condition que plusieurs fournisseurs jouent le jeu, ce processus de « coprescription ouverte » se prolonge jusqu'à la définition d'un cahier des charges techniques détaillant les besoins, à partir duquel l'acheteur organise une dernière consultation pour obtenir les offres commerciales. Le client exploite les solutions proposées par les différents fournisseurs pour reformuler ses besoins.

Le récit d'un dirigeant, fabricant de machines spéciales pour l'automobile, montre comment il tente d'équilibrer le jeu de pouvoir avec le client dans un processus de consultation : «Une rumeur me prévient d'un nouveau projet. Immédiatement j'essaie d'informer les bureaux d'étude client sur mes solutions techniques. J'invite les techniciens du BE, et tente de réorienter le futur " pré-cahier des charges de consultation" (PCC) en direction de ma solution technique. Ce PCC est ensuite distribué auprès des autres fournisseurs consultés. Plus le fournisseur essaie d'orienter le PCC en sa faveur, plus il doit proposer des idées nouvelles. Mais ces idées sont ensuite rendues publiques puisque le PCC est distribué... Donc il n'a pas toujours intérêt à avancer trop tôt les idées nouvelles.(...) Le client consulte en général davantage de fournisseurs que ceux qu'il pensait pouvoir retenir : il essaie de recueillir le plus grand nombre d'idées sur la question. Puis, sur la base des réponses, la discussion technique va s'affiner de plus en plus. Bien évidemment, j'insiste auprès du technicien sur les spécificités de mon offre: une machine plus rapide, avec moins de mouvement... On va aller très loin dans le détail. Les clients ont aussi leurs idées, leurs exigences. Ils se réunissent plusieurs fois pour définir leurs besoins. Chaque nouvel intervenant ajoute une idée, chaque nouvelle idée conduit à une variante supplémentaire. Les idées des fournisseurs 
s'ajoutent à leur tour. Le pré-cahier des charges prévoit l'ensemble des variantes possibles et les fournisseurs doivent proposer un coût pour chacune.(...)»

L'ouverture de la «coprescription » a une autre fonction : elle contribue aussi au maintien de la confiance du client vis-à-vis de ses fournisseurs privilégiés. Le client peut ainsi vérifier régulièrement que ces derniers ne tirent pas un bénéfice excessif de la relation de coopération qu'ils ont engagée.

Un acheteur évoque comment il a utilisé Renovutil pour concurrencer un fournisseur en place : «J'ai comparé Renovutil qui a fait une proposition (...). J'ai à coté le fournisseur actuel. Il se trouve que Renovutil est meilleur. J'aurais pu gagner du temps en ne faisant pas cette vérification du marché. C'est le plus beau dossier que je n'ai jamais vu en termes de maintenance. Il y a un inventaire exhaustif des équipements existants. Il y a des gammes de maintenance détaillées, c'est très professionnel comme travail contrairement à d'autres ; ils sont forts ! Avec nous, ils sont là, ils sont très réactifs ; dès que l'on besoin de ressources, ils sont là. Je les ai consultés sur un projet de modification : en une semaine, ils ont su nous répondre avec une enveloppe financière. »

Autrement dit, l'acheteur et les clients internes recherchent, par la «coprescription ouverte», les bénéfices de la compétition tout autant que les bénéfices d'une "relation de service », comme la mise à disposition des compétences des fournisseurs et leur investissement dans la singularisation de l'offre (l'adaptation aux besoins du client).

\section{La fixation des prix dans un processus de coprescription}

Les relations marquées par une forte coprescription ressemblent aux «marchés de prototypes », où les dimensions hors-prix sont prépondérantes dans le jeu concurrentiel (François, 2004). La fixation du prix ne fait pas l'objet d'un réel ajustement entre les clients et les fournisseurs, le prix s'établit sur une base conventionnelle partagée entre fournisseur et acheteur (barème, décomposition des coûts...). Le principal enjeu pour le fournisseur est de parvenir à justifier son prix. Un fournisseur qui s'écarterait trop des prix conventionnels et qui ne parviendrait pas à le justifier prendrait le risque d'affaiblir la relation de coopération avec le client interne et donc d'encourager ses interlocuteurs à choisir un autre mode de relation.

Le nouveau prestataire de maintenance Maintini, s'est investi dans l'ingénierie du contrat : c'est lui qui a proposé à son client de ne pas signer immédiatement un contrat au forfait, mais de passer par une période probatoire d'un an, avec une transparence totale sur les activités et les dépenses, «à livre ouvert». Un an plus tard, la négociation du contrat au forfait s'est faite sur une base d'informations partagées. Les dimensions hors-prix et prix ont été clarifiées...

Prenons les cas des prestations ponctuelles d'ingénierie de travaux de Renovbati et Renovutil : il s'agit d'offres spécifiques et le processus est assez rudimentaire : des barèmes permettent d'établir les coûts de main d'œuvre à partir du temps de travail prévu, le fournisseur consulte à son tour ses 
propres sous-traitants pour connaître leurs coûts. Puis il évalue la marge qu'il souhaite réaliser sur cette affaire, en fonction de la valeur de cette affaire pour l'ensemble de son activité, des incertitudes qui pèsent sur le projet, et du risque de ne pas obtenir l'affaire. Il tente aussi de se renseigner sur les propositions concurrentes : comment les concurrents définissent-ils leurs coûts et établissent-ils leur prix ? Le fournisseur teste ses prix régulièrement et récupère de l'information à chaque fois qu'il perd une affaire...

L'organisation d'une coprescription ouverte a aussi une influence sur la formation du prix : elle accroît le pouvoir de marché du client. Quand l'acheteur a réussi à conduire une coprescription ouverte et qu'il est parvenu à obtenir des offres techniques en grande partie substituables, il est en position de force pour organiser la négociation. Il peut étudier les décompositions des prix proposées par les fournisseurs, renégocier les offres techniques et les offres de prix. Un format imposé de devis est un moyen de mise en équivalence des offres et de comparaison des prix, tout en acceptant des offres techniquement différentes. Les offres alternatives sont donc des ressources essentielles dans la pratique de négociation de l'acheteur, bien plus intéressantes que la connaissance des prix « conventionnels».

\section{Pourquoi les fournisseurs continuent à collaborer avec leurs clients}

L'intensification de la compétition crée une incertitude pour les fournisseurs. Pourquoi les fournisseurs en place et les fournisseurs outsiders continuent-ils à coopérer si la contrepartie à leurs investissements devient aussi incertaine?

\section{Les pratiques de négociation des prix tentent de prolonger la logique coopérative}

La rationalisation de la coproduction n'est jamais complète et ne peut réduire totalement la dépendance du client vis-à-vis d'un fournisseur tout au long de l'exécution d'un contrat. Acheteur et fournisseurs en ont conscience, ce qui les conduit à modérer leur opportunisme (tant en amont de la signature du contrat, côté acheteur, qu'en aval, côté fournisseur) pour conserver une dynamique coopérative.

En effet, il existe tout un ensemble de pratiques de négociation qui permettent de combiner une attitude coopérative avec le maintien d'une menace de prendre un autre fournisseur. Quand l'acheteur a une préférence pour un fournisseur, en ce qui concerne les dimensions hors-prix, il l'informe du meilleur prix qu'il a obtenu par ailleurs. C'est ce que les acheteurs et commerciaux 
appellent le «last call », marque de confiance par l'acheteur. Elle encourage la coopération à venir $^{18}$.

«L'acheteur a le pouvoir final. S'il veut te prendre, tu le sauras, et il trouvera toujours le moyen d'habiller la décision. Il viendra te trouver et te faire comprendre que, si tu veux avoir l'affaire, il suffit de baisser de $4 \%$. Il ne le demandera pas aux autres fournisseurs et tu seras moins cher qu'eux. Il te donnera les indications pour que tu prennes l'affaire. S'il ne veut pas de toi, il ne te donnera pas d'indication ou des indications faussées. » (Dirigeant, fabricant de machines)

Cette pratique coopérative n'empêche pas des stratégies opportunistes à la marge : l'acheteur peut «bluffer» sur le prix du moins disant (mais il risque sa propre crédibilité). Réciproquement, côté fournisseur, une relation coopérative n'empêche pas non plus des stratégies opportunistes à la marge ${ }^{19}$. L'opportunisme reste modéré (Neuville, 1998) car il est de l'intérêt des partenaires de ne pas apparaitre comme « opportunistes » mais au contraire comme « arrangeants » et de ne pas mettre en jeu la relation de coopération et la réputation.

\section{La coprescription, un investissement risqué pour le fournisseur outsider}

Du point de vue du fournisseur, l'investissement dans la coprescription, en amont de la négociation du prix et de la signature du contrat, est un jeu risqué. Il s'agit bien d'un investissement, car il met à disposition du client disponibilité et compétence. Le risque demeure de ne pas être retenu. En effet, à la différence des marchés de prototypes étudiés par François (2004) ou des marchés des singularités de Karpik (2007), la coprescription dans un contexte industriel ne conduit pas nécessairement à une offre singulière où chaque fournisseur pourrait se différencier de façon déterminante.

\footnotetext{
${ }^{18}$ Quand les acheteurs sont conscients des interdépendances informelles, ils évitent des techniques de mise en concurrence qui exerceraient un pouvoir excessif sur les fournisseurs, comme par exemple l'enchère inversée. Selon cette technique, après avoir défini précisément le contenu de l'offre technique, l'acheteur invite les fournisseurs à faire des propositions de prix ; les fournisseurs sont automatiquement informés des propositions des autres et peuvent « sous-enchérir ». De l'avis des acheteurs interrogés, pour ne pas produire d'effet pervers, cette technique exige une formalisation initiale du besoin extrêmement précise. Sinon, cette technique risquerait d'exacerber des conflits d'intérêts au détriment de la coopération. Dans les cas analysé, pour cette raison là, cette technique n'est pas utilisée.

${ }^{19} \mathrm{Si}$ son client a besoin d'un composant en urgence, en dehors des dispositions contractuelles, le fournisseur lui « rend service » mais renégocie le prix à la hausse. L'occasion lui est donnée par le fait que la demande du client soit hors procédure ou hors délai. Le fournisseur argumente l'écart sur la base des coûts internes de réorganisation pour fournir la pièce à temps, coûts non vérifiables par le client.
} 
Comme le fournisseur en place, le fournisseur alternatif a besoin d'évaluer la probabilité de remporter l'affaire. Cependant, il n'a pas de relation privilégiée avec les clients internes. La probabilité est élevée qu'il joue un rôle de lièvre pour que le fournisseur déjà dans la place accepte de réduire ses coûts. Le fournisseur alternatif cherchera à comprendre la motivation réelle du client : le client interne est-il satisfait du fournisseur en place ? L'acheteur a-t-il besoin de lui pour rouvrir le jeu par rapport à un fournisseur en place qui «abuserait » de sa position ? Est-il prêt à s'engager sur un certain volume d'activité ? C'est pourquoi le fournisseur alternatif tente généralement d'obtenir de l'acheteur un engagement informel avant d'investir davantage dans la relation.

«Si un concurrent répond à l'appel d'offres, qu'il a été en amont du processus et que l'on arrive à le savoir, cela veut dire que l'on minimise nos chances de réussite; Un appel d'offres où 10 entreprises ont été consultées et où nous estimons nos chances de réussite à 1 sur 10, on se pose la question de savoir s'il n'y a pas un autre projet ailleurs, plus intéressant, parce qu'il nous permet de travailler en amont avant de s'engager. Cela dépend de la charge de travail et de ce que l'on sent...Là aussi, la phase de qualification est importante ; il s'agit de choisir son projet. » (chef de projet, Renovutil)

Ainsi, les pratiques de «coprescription ouverte » recherchent un certain équilibre entre mise en concurrence et engagement, équilibre qui se déplace, en faveur du fournisseur ou en faveur du client, au gré des stratégies des fournisseurs, des rapports de force économiques, des nécessités techniques, et des processus d'achat.

Les fournisseurs interrogés signalent une nouvelle tendance dans les pratiques des acheteurs : ces derniers imposent de plus en plus souvent un engagement sur un niveau de performance, ils demandent aux fournisseurs de prendre en charge l'ensemble des risques techniques et économiques du projet. Ces exigences laissent les fournisseurs perplexes : certains prennent le risque d'accepter ce type de contrat, mais ne prennent pas au sérieux les exigences contractuelles. Ils estiment que les incertitudes techniques sont inévitables, que leur interlocuteur technique les assumera en partie, et que l'acheteur acceptera, en « bonne entente », les avenants et les coûts supplémentaires. Ils prennent le risque d'une relation conflictuelle avec l'acheteur si celui-ci reste inflexible.

\section{La formation d'un pool de partenaires privilégiés}

L'organisation d'une « coprescription ouverte » est facilitée lorsque l'entreprise cliente dispose de plusieurs fournisseurs «insiders » sur lesquels l'acheteur a une responsabilité. Il peut les mettre en concurrence régulièrement sur de nouveaux projets. Pour les fournisseurs, contribuer à la coprescription est la règle du jeu pour conserver leur place et parce qu'ils anticipent des contreparties à moyen terme. 
«L'acheteuse m'explique - Je te challenge là dessus, on te fait toujours confiance mais là j'ai mis une autre boite dans la boucle parce que globalement... - En fait je savais qu'elle avait vu le consultant concurrent, c'était lui quoi. Il n'y avait pas d'autre solution, il était déjà passé avant. Et moi j'ai fait une proposition, comme d'habitude, 26 pages, bien détaillées, on a compris son besoin. (...) Elle me rappelle : «OK, c'est bon, je viens de voir mon boss ». (Commercial, cas Informa)

Constituer un tel pool et en avoir le contrôle, suppose une centralisation des achats et une capacité à imposer aux différentes usines le choix des fournisseurs. Autrement dit, l'acheteur cherche à substituer aux liens entre les usines et leur fournisseur privilégié, un lien entre luimême et un pool de partenaires substituables. Les fournisseurs qui souhaitent accroître leur part de marché accepteront d'investir. L'acheteur se perçoit comme engagé, informellement, vis-àvis des fournisseurs qui jouent son jeu. Cependant cette stratégie est tributaire de la coopération des usines.

Quand la «coprescription»suppose de très gros investissements, comme le développement d'un prototype $\mathrm{e}^{20}$, le client contribue généralement à son financement. La «coprescription ouverte » n'est pas possible puisque doubler les investissements dans un prototype est trop coûteux. Le fournisseur bénéficie alors d'une position avantageuse. L'acheteur tente de circonscrire cet avantage en organisant la concurrence en amont de l'investissement dans le prototype, mais il doit accepter que la performance ne puisse être définie totalement en amont et que la coprescription se prolonge après signature du contrat. L'acheteur limite l'opportunisme « post-contractuel » en jouant sur les nouveaux projets.

Ainsi, l'idée de «coprescription ouverte » comprend une contradiction implicite : elle suppose un investissement significatif des fournisseurs tout en les menaçant de ne pas être retenus. Dans ce contexte, la «coprescription ouverte» au sein d'une «niche sociale » (Lazega, Mounier, 2002) semble être la configuration la plus équitable et la plus incitative : les clients internes comme les acheteurs peuvent jouer sur un volet d'affaires assez large pour organiser à la fois la compétition et la rétribution des investissements en coprescription. Ce compromis n'est pas non plus à l'abri d'une intensification de la concurrence ou d'une ouverture à des acteurs extérieurs à cette niche.

\footnotetext{
${ }^{20}$ Situation rencontrée dans le cas Electro.
} 


\section{Comment l'acheteur convertit le client interne aux vertus du marché, comment le client interne modère les ambitions de l'acheteur}

Pour la standardisation des besoins internes, la sélection, l'accompagnement des fournisseurs alternatifs, et enfin la coprescription ouverte, le travail de l'acheteur est dépendant de la coopération des clients internes. Et ce, pour de multiples raisons: ils détiennent des compétences utiles dans le choix et le contrôle des fournisseurs, et jouent souvent un rôle de «technical gate keeper»(Pettigrew, 1975), ils contribuent aux décisions et à leur mise en œuvre. Ce degré de dépendance varie donc selon le niveau de compétence technique exigé par l'achat et la répartition des compétences techniques entre acheteur et client interne. Il varie aussi selon les procédures décisionnelles. Dans l'ensemble des cas étudiés, cette dépendance est significative.

Or il n'y a pas de raison pour qu'acheteurs et clients internes perçoivent avec le même degré d'urgence ou d'importance la nécessité de changer ou de mettre en concurrence un fournisseur. En effet, ils ne sont pas inscrits dans la même structure d'incitation, ne poursuivent pas les mêmes objectifs, ne formulent pas les mêmes critères de jugement. La décision et l'organisation d'un changement de fournisseur supposent un alignement de l'acheteur et des clients internes sur une stratégie commune.

\section{L'agenda de l'acheteur est rarement en phase avec l'agenda des clients internes}

Les acheteurs interviewés présentent leur activité comme inscrite dans différentes politiques d'achat $^{21}$ : la centralisation des achats, la participation des acheteurs aux projets, le développement des achats dans les pays à «bas coûts». Les politiques d'achats jouent un rôle prépondérant comme déclencheur de l'action de l'acheteur. Côté client interne, le souhait de voir un fournisseur mis en concurrence répond à des enjeux opérationnels de qualité, de compétence, et plus rarement, de budget.

Autrement dit, les «agendas » des deux fonctions ne sont pas nécessairement synchronisés. Un jugement négatif par les clients internes sur un fournisseur (en termes de qualité ou de réactivité) ne signifie pas que les acheteurs accepteront d'investir dans une procédure de changement. Certains clients internes interrogés rapportent (de façon ironique) que, lorsqu'ils

\footnotetext{
${ }^{21}$ Les politiques achats sont souvent le reflet des évolutions institutionnelles de la fonction : la fonction Achats se développe grâce aux influences des consultants et forums professionnels qui donnent aux acheteurs les arguments pour accroître la légitimité de leurs actions.
} 
signalent des difficultés récurrentes avec un fournisseur en termes de qualité ou de délais, «l'acheteur utilise ce type d'argument pour faire baisser les prix, mais ne répond pas à nos besoins, ne change pas le fournisseur, sauf si les problèmes deviennent critiques». Réciproquement, les acheteurs se plaignent volontiers des nombreuses sollicitations des clients internes, qui leur demandent de «faire pression sur les fournisseurs » qu'ils jugent responsables des moindres problèmes de qualité.

Un décalage trop important entre les agendas des clients internes et de l'acheteur peut affaiblir la légitimité de l'acheteur aux yeux des clients internes et donc la coopération de ces derniers. Réciproquement, quand l'acheteur bénéficie d'une marge d'appréciation dans la définition de ses priorités et qu'il exploite cette marge de manœuvre pour se rapprocher de l'agenda des clients internes, sa légitimité est renforcée.

\section{L'ouverture de la coprescription remet en question les frontières internes}

Les acheteurs se sont longtemps positionnés sur des achats de fournitures où la qualité était standardisée et le service quasi absent, et ont toujours mis en avant les pratiques de « réduction des coûts » pour caractériser leur fonction. Compte tenu de cet héritage historique, les clients internes ne leur reconnaissent pas la compétence suffisante pour prendre en compte les caractéristiques hors-prix. Les acheteurs ont été longtemps exclus des achats dit «techniques » (machines, investissements...) ou des achats de prestations de service et plus généralement, ils ont été exclus du processus de « coprescription».

Le processus de coprescription se matérialise principalement dans la rédaction du cahier des charges techniques. Pour organiser une «coprescription ouverte», les acheteurs cherchent à accompagner les clients internes dans la rédaction de celui-ci. Ils veillent à ce que le cahier des charges soit précis sur les fonctions attendues du produit, mais le plus ouvert possible sur les solutions, afin de permettre à plusieurs fournisseurs de proposer des solutions.

Or, le cahier des charges est traditionnellement le domaine réservé des techniciens, chez le fournisseur et chez le client. Il est rédigé dans le cadre d'une culture technique et exige pour sa compréhension la maitrise de cette culture. Un acheteur qui souhaite ouvrir la « coprescription » doit donc être en mesure de dialoguer avec le client interne et les fournisseurs, et de se réapproprier cet objet ${ }^{22}$.

\footnotetext{
${ }^{22}$ La capacité à interpréter et discuter le contenu d'un cahier des charges ou d'un plan est de plus en plus exigée des acheteurs quand il s'agit d'achats techniques. Pour ce type d'achat, les entreprises privilégient des acheteurs ayant
} 


\section{La faible coopération interne exacerbe la tension entre le prix et les critères hors prix dans la relation avec les fournisseurs}

La relation entre acheteur et client interne, malgré une sophistication des instruments de suivi, de procédures de décision, et la plus grande proximité des incitations, est souvent entachée de suspicion. Acheteur et client interne s'accordent de façon tacite sur un partage des tâches : au client interne, les dimensions hors prix de l'achat, à l'acheteur, la négociation du prix.

Le maintien de cette division du travail est autant intentionnelle que non intentionnelle : en effet, les acheteurs travaillent souvent avec des données incomplètes, comme des benchmarks de prix, et ils ne souhaitent pas exposer au regard des clients internes leurs propres incertitudes. De même, les clients internes ne peuvent pas formaliser plus précisément leurs besoins, faute de temps et de compétence. Cependant, les informations sur les prix font aussi partie de la «zone d'incertitude » (Crozier, Friedberg, 1977) préservée par l'acheteur et sur laquelle il construit son pouvoir. Réciproquement, les clients internes conservent leur propre «zone d'incertitude»: leur besoin technique ou leur évaluation des fournisseurs.

« (à propos de la formalisation du Cahier des Charges) Ben c'est le gros point faible du projet. S'il me dit qu'un fournisseur ne tient pas la route techniquement et donc qu'il ne veut pas travailler avec lui, moi je ne peux pas aller à l'encontre de ça. Je n'ai pas trop de pouvoir là dessus comme il n’y a pas de Cahier des Charges. En plus, les fournisseurs ne répondent pas sur les mêmes bases. Alors pas facile si on veut comparer des choses comparables. » (Acheteur, cas Renovutil)

Les conséquences de cette faible coopération peuvent être aggravées par les procédures d'achat qui imposent un enchaînement séquentiel des étapes de définition des besoins, puis de consultation des fournisseurs. Une véritable exploration des alternatives suppose que l'acheteur puisse renégocier les besoins internes et explorer avec le client interne de nouvelles possibilités techniques avec de nouveaux fournisseurs... Autrement dit, l'acheteur ne peut rester prisonnier d'une démarche trop séquentielle. Il a aussi besoin de temps. Or les clients internes sont euxmêmes contraints par le déroulement des projets et ne prévoient pas toujours un délai suffisant. L'exploration des alternatives est alors impossible.

La faible coopération entre acheteurs et clients internes, l'absence d'exploration des opportunités techniques et économiques exacerbent le conflit entre la prise en compte des

une culture technique significative : en général ils possèdent soit une formation de technicien complétée par une expérience technique et un diplôme de management des achats. 
caractéristiques hors prix et celle du prix. Le fournisseur fait face à une organisation « schizophrène ».

\section{La sensibilité des clients internes vis-à-vis des coûts d'achat s'accrô̂t avec la pression budgétaire}

La sensibilité du client interne au prix d'achat dépend du cadre organisationnel dans lequel il est placé, et en particulier l'organisation budgétaire. En principe, les coûts des achats sont imputés sur le budget du client interne (l'acheteur dispose du « mandat » pour acheter, mais ce n'est pas lui qui est le bénéficiaire du bien acheté) et le client interne est généralement incité par sa propre hiérarchie à minimiser ses coûts globalement (main d'œuvre, achat, investissement) pour améliorer la profitabilité de son activité. Dans ce cas, il a intérêt à collaborer aux actions de réduction des coûts engagés par les acheteurs, et a fortiori, aux actions permettant la mise en concurrence des fournisseurs.

Cependant, dans la majorité des cas étudiés, l'organisation budgétaire est trop dispersée pour être incitative : les coûts d'achat d'investissement, les coûts d'achat en fonctionnement et les ressources humaines internes relèvent de budgets différents. S'ils ne contrôlent pas les budgets d'achat, les clients internes n'ont pas intérêt à accepter un fournisseur «moins disant»: les insuffisances d'un fournisseur moins performant techniquement pèseront directement sur leur activité, et ils ne tireront pas bénéfice d'un prix d'achat serré. Si les clients internes ont le sentiment que leurs propres marges de manœuvre en fonctionnement (ressources humaines internes, budget disponible pour financer des avenants) sont limitées, ils défendront le choix d'un fournisseur « mieux disant», qui donne des garanties de compétence et de disponibilité. Ni l'acheteur ni le client interne ne raisonne en coût complet : la grande complexité du calcul les décourage. Ils se résignent à travailler avec des critères hétérogènes de prix et de qualité de prestation.

\section{Les apprentissages croisés facilitent l'alignement des stratégies}

Lorsque sont réunies plusieurs conditions favorables (structure budgétaire incitative, investissement de l'acheteur dans les démarches de résolution de problème...) une boucle vertueuse d'apprentissage et de confiance s'engage naturellement entre acheteurs et client internes.

Comme l'explique l'acheteur dans le cas de Renovbati : «Au début c'était difficile. Et en avançant, dans le temps, sur le projet, ben il (le client interne) s'aperçoit qu'il y a un intérêt à ce que je travaille avec lui et depuis, en fait il m'intègre quasiment partout alors qu'à l'origine je devais intervenir que sur les gros corps de métiers. » Le client interne confirme : «Je pense que 
nous on apporte plus de technique à l'acheteur et je pense que c'est un bon compromis entre les deux. »

Cette boucle d'apprentissage est tout d'abord alimentée par la présentation de l'étendue des opportunités par l'acheteur (il s'appuie sur son travail préalable de prospection des marchés amont). La critériologie qui permet de comparer les différentes offres est mobilisée comme un instrument de dialogue entre acheteurs et clients internes. De même, en organisant l'information sur les besoins, sur les offres et sur les fournisseurs, les acheteurs structurent la délibération collective.

Cette boucle d'apprentissage peut s'engager aussi dans des conditions moins favorables. Soit l'acheteur possède suffisamment de compétences techniques, soit il peut mobiliser un bon réseau d'experts internes et externes pour constituer son dossier technique et économique et le soumettre aux clients internes. Il oblige ainsi les différents acteurs internes à se justifier et à révéler leurs informations et leurs jugements. Plus le débat technique est alimenté, plus l'acheteur étoffe son dossier. Il est alors de plus en plus difficile pour les clients internes de le contester.

La coopération entre achat et client interne n'est pas sans conséquence sur la mise en concurrence : leur présence conjointe dans la négociation permet de prendre en compte autant les dimensions techniques que le prix et accroit sensiblement le pouvoir de négociation sur le fournisseur.

«En fait pour que ça marche avec le fournisseur, il faut que ça marche avec le prescripteur. Si tu n'as pas une relation étroite, de confiance avec ton prescripteur, là tu vas au clash et ce n'est même pas la peine d'essayer de faire quoi que ce soit avec le fournisseur. Sur ce chantier ça se passe bien mais sur d'autres ça a été beaucoup plus difficile, le responsable du chantier faisait son truc de son côté, ne me communiquait rien et en réunion fournisseur moi j'avais l'air d'une potiche.» (Acheteur, cas Rénovutil)

Une plus grande cohérence interne n'empêche pas que l'acheteur et le client interne puissent avoir intérêt à différencier leur rôle quand il s'agit d'interagir avec les fournisseurs : ainsi, si l'acheteur souhaite peser sur le prix, par exemple face à un fournisseur en place, il organise un « rituel marchand» (Weber, 2000) dont il tient le rôle principal, il impose une compétition sur les prix et mettra entre parenthèses les liens d'interdépendance avec les clients internes. De leur côté, les clients internes se gardent bien de participer à ce rituel pour conserver la qualité des relations interpersonnelles qui facilitent les échanges avec le fournisseur en place. Ainsi, acheteurs et clients internes peuvent aussi jouer du fait d'être deux afin d'établir des formes d'interaction diversifiées avec les fournisseurs, alterner mise à distance et engagement. 


\section{Le conflit est aussi un moyen de rendre visible les coûts internes induits et de solliciter un arbitrage}

La résistance des clients internes à un changement de fournisseur est un moyen de faire reconnaître les coûts induits et les risques engendrés par le changement, afin de ne pas être exposés en cas de dégradation de la qualité ou de prestation non conforme aux attentes.

Pour gagner en légitimité et en influence auprès des clients internes, certains acheteurs se concentrent sur les activités où les clients internes ne sont pas satisfaits du fournisseur en place. L'acheteur acquiert son influence par un glissement de tâches qui le conduit à assumer en partie des réorganisations en principe prises en charge par les clients internes. C'est à cette condition qu'il peut conduire son projet de changement de fournisseurs. Ce glissement est d'autant plus aisé que les structures organisationnelles sont souples, que l'acheteur a lui-même les compétences techniques adéquates. Nous avons constaté un fort investissement des acheteurs dans le processus d'évaluation, de test et de qualification des produits issus des nouveaux fournisseurs : dans le cas Color, les problèmes de non qualité rencontrés par les lignes de production ont été pleinement assumés comme la conséquence du changement de fournisseur et les équipes ont été soutenues et valorisées dans leur capacité à les surmonter.

Si le conflit s'aggrave, acheteur et clients internes sollicitent un arbitrage hiérarchique. L'acheteur sollicitera l'arbitrage dans le cas d'un programme de réduction de coûts solidement argumenté et ayant une forte légitimité. Les clients internes peuvent faire valoir les conséquences négatives pour leur activité et obtenir des ressources en cas de dégradation de la qualité ou de la performance.

Néanmoins, la centralisation des achats entraîne plutôt une forte séparation entre la hiérarchie des acheteurs et la hiérarchie technique et industrielle. Les arbitrages ne peuvent donc être effectués qu'à un haut niveau de l'organisation, qui présente toujours des risques importants pour ceux qui l'invoquent. Acheteurs et clients internes ont donc parfois intérêt à éviter l'arbitrage et à privilégier les arrangements. Le meilleur arrangement est l'évitement : l'acheteur révise son agenda, privilégie les usines qui le sollicitent, délaisse les usines qui coopèrent insuffisamment. Alors que l'acheteur dispose, avec la centralisation des achats, d'un formidable levier pour mettre en compétition ses fournisseurs, son éloignement organisationnel vis-à-vis des clients internes conduit à un évitement réciproque favorable au maintien des fournisseurs en place!

\section{Conclusion}

Notre recherche propose une investigation qualitative des jeux de pouvoir et des dynamiques de changement entre clients et fournisseurs, quand les fournisseurs sont investis dans une « relation 
de service » auprès de leur client. Dans les cas étudiés, les fournisseurs assument, sans que cela ne soit formalisé, un grand nombre de tâches en aide aux clients internes, tant dans l'expression de leurs besoins en amont que dans le quotidien des prestations ou des livraisons. En échange, ils essaient d'obtenir une certaine stabilité de la relation, les clients n'ayant pas vraiment intérêt à travailler avec d'autres fournisseurs qui ne feraient pas les mêmes investissements, qui n'auraient pas accumulé les mêmes apprentissages ou qui n'offriraient pas les mêmes garanties.

Néanmoins, nous observons que les clients maintiennent une forte compétition entre fournisseurs : les relations sont rarement exclusives et la stabilité n'est jamais assurée. Cette compétition est le principal levier de pouvoir du client et est principalement utilisée dans la maitrise des prix.

Pour examiner comment les clients combinent collaboration et compétition, nous avons adopté une stratégie de recherche originale: enquêter auprès d'acheteurs, de clients internes et de fournisseurs sur des processus de choix et de changement de fournisseurs. Nous avons pris le parti d'étudier des situations «critiques », où les clients internes exprimaient des besoins de « relation de service » et des craintes de l'affaiblissement de celle-ci avec l'action de l'acheteur.

Ainsi, nous montrons que l'acheteur développe une capacité à maintenir une compétition entre fournisseurs en rendant possible (moins coûteuse et moins risquée) la substitution entre fournisseurs. Des pratiques de «cadrage » (Callon, 1999) réduisent ou réintègrent dans le jeu de la compétition la prolifération des échanges informels : par exemple, la rationalisation et la standardisation des besoins réduisent l'ampleur des ajustements informels autour des flux de produits et de leur qualité (sans les éliminer tout-à-fait). Les acheteurs travaillent aussi à la qualification des offres afin de limiter leur dimension singulière (Karpik, 2007) : la formalisation de critères permet de comparer l'engagement des fournisseurs, l'implication de plusieurs fournisseurs dans la définition des besoins et des solutions techniques (que nous avons appelé « coprescription ouverte ») permet de disposer d'alternatives comparables. Les acheteurs répondent à la stratégie de captation par les fournisseurs en place par une maîtrise de la singularisation ${ }^{23}$. Comme il reste une part irréductible d'incertitude et de singularité, l'acheteur cherche, tout au long des processus de négociation, à conserver une atmosphère coopérative

\footnotetext{
${ }^{23}$ Autrement dit, il existe une gradation dans le processus de singularisation et les attachements qu'il provoque : même si la singularisation existe sur tous les marchés (Callon et al. 2000), elle provoque plus ou moins d'irréversibilité et de dépendance entre acteurs, et donc a des effets plus ou moins prononcés sur la configuration du marché et l'intensité de la concurrence.
} 
avec le fournisseur, de façon à ce que ce dernier soit toujours encouragé à contribuer par la relation de service aux besoins non formalisés par le client.

Cette recherche montre l'intérêt d'une analyse de l'activité des acheteurs et pose un certain nombre de questions nouvelles qui pourraient faire l'objet d'investigations complémentaires. Aussi, nous avançons que le travail des acheteurs, étayé par la segmentation, la révision des cahiers des charges, l'usage de formats de devis et procédure d'appel d'offre, leur permet d'accéder à un plus grand nombre d'offres alternatives, de les caractériser et de les comparer... L'acheteur, ses méthodes et ses instruments contribuent à la construction du client comme agent économique calculateur. Une observation ethnographique de ces pratiques et de l'usage de ces instruments permettraient de discuter les conditions dans lesquelles ces instruments accroissent la « capacité de calcul» (Callon, Muniesa, 2005) de l'entreprise cliente.

Notre travail suggère que la réintroduction du marché passe par des investissements relationnels diversifiés : 1'acheteur a besoin de maintenir et développer un réseau de relations avec des liens un peu «moins engageants» que les liens existants, pour construire les alternatives et la comparaison. L'acheteur trouverait l'essentiel de sa légitimité et de son pouvoir dans sa capacité à nouer des relations avec des acteurs a priori plus « distants » et n'ayant pas de lien entre eux, autrement dit, à se placer dans des trous structuraux des réseaux d'échanges interindustriels (Burt, 1992). Une analyse plus approfondie et systématique des réseaux relationnels des acheteurs (et en particulier de leur dimension internationale) et une comparaison avec les réseaux relationnels des «clients internes » permettraient d'approfondir le rôle des acheteurs dans la mondialisation. Le rôle des acheteurs pourrait aussi être approfondi, dans une perspective institutionnaliste (Fligstein, 1991), par une enquête systématique pour mesurer la montée en puissance de la fonction achat sur la période récente, l'extension de son rôle organisationnel, et les effets en termes de globalisation des achats.

Enfin, notre enquête met en valeur les tensions entre acheteurs et clients internes. En effet, les efforts des fournisseurs en place pour développer des synergies bénéficient aux clients internes, alors que la mise en concurrence (en particulier sur le prix) bénéficie plutôt aux acheteurs. Les quelques cas étudiés suggèrent que le conflit est exacerbé par les politiques achats de réduction de coût ou de changement de fournisseur quand elles sont en décalage avec les besoins des clients internes. Il n'y a pas une rationalité unique qui régule engagement et concurrence, mais différentes rationalités qui, au gré des changements organisationnels et des arbitrages managériaux, dominent l'une après l'autre. Nous montrons que le client parvient à se comporter comme agent économique qu'au prix de l'alignement des acheteurs et acteurs internes sur une même stratégie, situation qui est davantage l'exception que la règle. 
Il est utile de préciser les limites de validité de nos résultats. Pour comparer plusieurs cas différents, nous avons fait le choix de réduire la complexité des jeux internes chez le client et la diversité des acteurs impliqués en mettant en valeur l'opposition entre acheteur et «client interne $\gg .$. Une autre stratégie de recherche pourrait au contraire valoriser cette diversité, au prix de ne pas pouvoir conduire un travail comparatif. Par ailleurs, la méthode d'enquête par entretiens approfondis auprès des différents acteurs impliqués autour de situations précises n'a pas facilité pas l'exhaustivité des répondants. Nous avons été confrontés à des enjeux de confidentialité stratégique mais aussi de conflits entre acteurs. D'autres techniques d'enquête plus anonymes contournent ce problème mais ne permettent pas d'obtenir un croisement des discours, ni d'obtenir une information qualitative suffisamment riche, ni de construire de véritables études de cas ${ }^{24}$.

Par ailleurs, nos résultats dépendent aussi de la nature des activités concernées. Il s'agit d'activités intenses en coopération mais qui connaissent une certaine maturité technique (repérable dans le fait que les offres sont interchangeables) et des marchés qui sont stables, voire en régression ${ }^{25}$. Cette recherche pourrait être prolongée vers des activités comme la soustraitance industrielle de pièces simples ou d'activités de montage qui présentent une maturité technique plus avancée encore et une moindre incertitude sur la qualité et les flux. Notre recherche suggère que la substituabilité et la mise en concurrence s'y organisent plus facilement encore.

L'intensification de la compétition pourrait être mieux mesurée. Les cas étudiés montrent que l'exercice de la compétition reste confiné à un cercle limité de partenaires, parce que l'interchangeabilité reste lourde à organiser. De plus, les «outsiders» doivent consentir à un ticket d'entrée, un investissement relationnel qui tient aux activités étudiées, exigeantes en « relation de service » et en apprentissage. Nos interlocuteurs fournisseurs évoquent néanmoins une forte incertitude sur l'obtention du marché et une forte pression sur les prix lors de la négociation quand il existe au moins deux ou trois compétiteurs. Cette relation asymétrique est probablement liée au contexte économique: dans la majorité des cas étudiés (service à

\footnotetext{
${ }^{24}$ Des entretiens approfondis complémentaires auprès de chefs de projets ou dirigeants confrontés à des situations comparables nous ont permis de consolider notre analyse.

${ }^{25}$ L'intensité concurrentielle y est souvent plus forte que dans des secteurs où la recherche et l'innovation tiennent une place importante, mais qui connaissent aussi des marchés en expansion (secteurs où la plupart des enquêtes sur les réseaux d'entreprises ont été conduites).
} 
l'industrie), les prestataires poursuivent des stratégies de croissance sur un marché qui ne progresse plus beaucoup.

Enfin, il est difficile d'évaluer toutes les conséquences internes des stratégies d'intensification de la compétition. Les cas étudiés, quand la concurrence a pu s'exercer, sont considérés comme des réussites pour l'ensemble des acteurs chez le client, passée une période d'ajustement souvent éprouvante ${ }^{26}$. Mais ce résultat ne peut être généralisé : le client interne n'est pas à l'abri d'une dégradation de la relation de service, d'une détérioration de la situation des fournisseurs et d'une intensification du travail, en interne, pour compenser. Notre enquête n'exclue pas non plus l'existence de ruptures radicales avec une dégradation de l'offre de service du fournisseur (Emberson, Storey, 2006). Une analyse longitudinale approfondie d'une stratégie d'achat, en partenariat avec un donneur d'ordre (avec un accès aux contrats et à la comptabilité analytique) permettrait d'évaluer plus précisément la performance économique de ce type de stratégie.

\footnotetext{
${ }^{26}$ Y compris dans le cas Color, emblématique d'un passage en force, avec le remplacement d'une offre singulière à une fourniture standardisée, qui a eu pour effet une «réintégration» chez le client de compétences déléguées jusqu'alors au fournisseur. La qualité de l'accompagnement a eu un effet décisif sur l'acceptation du changement.
} 
AFNOR, 1990. - Outils de management - lignes directrices pour les achats et les approvisionnements. FD X50-128.

Azimont, F., Cova, B. \& Salle, R., 1999. - «Vente de solutions et marketing de projet : une même recherche d'intimité client pour une construction conjointe de l'offre et de la demande », Revue Française du Marketing, n 173-174.

Berger S., 2006, - Made in monde, Les nouvelles frontières de l'économie mondiale - Seuil

Berry M., 1983. - Une technologie invisible, L'impact des instruments de gestion sur l'évolution des systèmes humains, Cahier du Centre de Recherche en gestion.

Boussard V., 2001. - «Quand les règles s'incarnent. L'exemple des indicateurs prégnants », Sociologie du Travail, Volume 43, 4, 2001, p. 533-551

Burt, Ronald S. (1992), Structural Holes, The Social Structure of Competition, Cambridge, MA: Harvard University Press.

Callon, M., 1999. «La sociologie peut-elle enrichir l'analyse économique des externalités ? essai sur la notion de cadrage-débordement » in Innovations et performances : approches interdisciplinaires, Paris, Editions de l'Ecole des Hautes Etudes en Sciences Sociales

Callon M., Meadel C., Rabeharisoa V., 2000. - «l'économie des qualités », Politix, 13 (52), pp. 211-239

Callon, M., Muniesa, F., 2005 .- «Economic Markets as Calculative Collective Devices », Organization Studies, Vol. 26, No. 8, 1229-1250

Calvi R.,2000.- Le rôle des services achats dans le développement des produits nouveaux : une approche organisationnelle Finance Contrôle Stratégie - Volume 3, Nº 2, juin, p. 31 - 55.

Chantelat P., 2002. - «La nouvelle sociologie économique et le lien marchand : des relations personnelles à l'impersonnalité des relations », Revue française de sociologie, 43, 3, pp. 521556.

Cochoy F., Dubuisson-Quellier S., 2000. - «Introduction. Les professionnels du marché : vers une sociologie du travail marchand », Sociologie du travail, 42, 3, pp. 359-369

Combes M.-C., 2002. - « La compétence relationnelle, une question d'organisation », Travail et emploi $\mathrm{n}^{\circ} 92$, octobre,

Comet C., 2007. - «Capital social et profits des artisans du bâtiment : le poids des incertitudes sociotechniques », Revue française de sociologie 48, 1, p. 67-91. 
Crozier M., Friedberg E., 1977. - L'acteur et le système, Seuil, Paris

Cyert, R. M., J. G. March. 1963. A Behavioral Theory of the Firm. Prentice Hall, Englewood Cliffs, NJ.

De Bandt J. Gadrey J., 1997. - Marchés de service, relations de service, Paris, Ed. CNRS

Dubuisson-Quellier S., 1999. - «Le client, le prestataire et le consommateur. Sociologie d'une relation marchande », Revue Française de Sociologie, 40, 4.

Dyer, J.H., Nobeoka, K., 2000. - «Creating and Managing a high performance knowledgesharing network: the Toyota Case », Strategic Management Journal, Special Issue March, pp. 345-369

Eisenhardt K.M., Graebner M. E., 2007. - « Theory Building From Cases: Opportunities And Challenges », Academy of Management Journal, 50, 1, 25-32.

Emberson, C., Storey, J., 2006. - «Buyer-supplier collaborative relationships: Beyond the normative accounts », Journal of Purchasing \& Supply Management 12, 236-245

Fligstein, N. 1991 "The structural transformation of American industry: An institutional account of the causes of diversification in the largest firms, 1919-1979." In W. W. Powell and P. J. DiMaggio (eds.). The New Institutionalism in Organizational Analysis: 311-336. Chicago: University of Chicago Press

François P., 2004. - «Prototype, concurrence et marché : le marché des concerts de musique ancienne », Revue Française de Sociologie , 45-3, 2004, pp. 529-559

Friedberg, E., 1993. - Le Pouvoir et la Règle. Dynamique de l'action organisée, Seuil, Paris.

Friedman, T. L. 2005. - The World Is Flat, Farrar, Straus and Giroux, New York:

Gadde, L.-E., Huemer, L., Håkansson, H., 2003. - «Strategizing in industrial networks » Industrial Marketing Management, 32, 5, p357-366

Garcia M.-F., 1986. - « La construction sociale d'un marché parfait : le marché au cadran de Fontaines-en-Sologne », Actes de la recherche en sciences sociales, 65, pp. 2-13.

Granovetter M., 1973, «The Strength of Weak Ties », American Journal of Sociology, vol. 78, $\mathrm{n}^{\circ} 6$

Gulati R., Sytch M. 2007. - "Dependance Asymmetry and Joint Dependance in Interorganizational relationship : Effect of Embeddedness on a Manufacturer's Performance in Procurement Relationship", Administrative Science Quarterly, 52 (2007): 32-69 
Hatchuel, A., 1997. - « Modèles de service et activités industrielles, la place de la prescription » in De Bandt J. Gadrey J., Marchés de service, relations de service, Paris, Ed. CNRS, p. 63-84,

Holm, D. Blankenburg, Eriksson, K., 1999. - « Creating value through mutual commitment to business network relationships », Strategic Management Journal, 20, 5, pp. 467-487

Johnston, W. J., Bonoma, T. V., 1981. - «The Buying Center: Structure and Interaction Patterns », Journal of Marketing, 45, 3, pp. 143-156

Karpik L. 2007. - L'économie des singularités, Paris, Gallimard

Langley, A. - 1999, "Strategies for Theorizing from Process Data." Academy of Management Review 24(4): 691.

Lazega, E. et Mounier, L.. - 2002 "Interdependent entrepreneurs and the social discipline of their cooperation: The research program of structural economic sociology for a society of organizations", in O.Favereau et E.Lazega (eds), Conventions and Structures in Economic Organization: Markets, Networks, and Hierarchies, Cheltenham, UK: Edward Elgar Publishing, pages 147-199).

Lonsdale, C., Watson, G., 2005. - «The internal client relationship, demand management and value for money: A conceptual model », Journal of Purchasing \& Supply Management 11, pp. $159-171$

Mariotti F., 2004 - «Entreprise et gouvernement : à l'épreuve des réseaux », Revue française de sociologie., 45-4, 711-737

Mariotti F., 2005. - Qui gouverne l'entreprise en réseau, les Presses de Sciences Po.

Meyer John W., Rowan Brian, 1977. - «Institutionalized Organizations: Formal Structure as Myth and Ceremony », The American Journal of Sociology, 83, 2 , pp. 340-363

Neuville, J.-P., 1998.- « La tentation opportuniste. Figures et dynamiques de la coopération interindividuelle dans le partenariat industriel », Revue Française de Sociologie, 39, 1, pp. 71 à 103

Pettigrew A.M., 1975. - « The Industrial Purchasing Decision as a Political Process », European Journal of Marketing , 9, 1

Podolny, J. M., 1994.- "Market uncertainty and the social character of economic Exchange", Administrative Science Quarterly, 39, 458-483.

Podolny, J. M., Stuart T.E. and Hannan M.T., 1996. - “ Networks, knowledge, and niches, competition in the worldwide semiconductor industry, 1984 à 1991", American Journal of Sociology, 102, 659-689. 
Powell, Walter W., 1990. - "Neither market nor hierarchy: network forms of organization" in L.L. Cummings and B. Shaw (eds), Research in Organizational Behavior, vol. 12, Greenwich, CT: JAI Press.

Powell, W.W., Koput K.W. and Smith-Doerr L., 1996.- "Interorganizational collaboration and the locus of innovation: networks of learning in biotechnology", Administrative Science Quarterly, 41, 116-145.

Ranjay; G., Gargiulo, M., 1999. - «Where do Interorganizational Networks Come From?» American Journal of Sociology, 104, 5, p1439-1493

Segrestin D., 1997, - «L'entreprise à l'épreuve des normes de marché - Les paradoxes des nouveaux standards de gestion dans l'industrie », Revue française de sociologie, 38 (3), pp. 553585

Star, S., J. Griesemer. 1989. - « Institutional economy, "translations" and boundary objects: amateurs and professionals in berkeley's museum of vertebrate zoology 1907-39», Soc. Stud. Sci. 19(9) 387-420.

Strauss, A. L. \& Corbin, J., 1990. - Basics of qualitative research: grounded theory procedures and techniques. Newbury Park : Sage

Swedberg R., 1994. - Une histoire de la sociologie économique, Paris, Desclée de Brouwer Trompette P., 2005. - «Une économie de la captation. Les dynamiques concurrentielles au sein du secteur funéraire », Revue Française de Sociologie, 46-2, pp. 233-264.

Uzzi B., 1997. - «Social structure and competition in interfirm network, the paradox of embedness », Administrative Science Quarterly, 42 (1), 35-67

Uzzi, B., 1999.- "Embeddedness in the making of financial capital: how social relations and network benefit firms seeking capital”, American Sociological Review, 64, 481-505.

Weber M., 1922. - Économie et société, Paris, (traduit et réédité en 2005, pocket)

Weber F., 2000. - «Transactions marchandes, échanges rituels, relations personnelles, une ethnographie économique après le grand partage », Genèses 41, déc, pp. 85-107

Williamson O. E., 1985. - The economic institutions of capitalism, New York, Free Press [Trad. française : Les institutions de l'économie, Paris, InterÉditions, 1994].

White, H., 1981.- "Where do markets come from?”, American Journal of Sociology, 87, 517-47. Whitford Josh, 2005.- The New Old Economy: Networks, Institutions, and the Organizational Transformation of American Manufacturing, Oxford University Press. 
Tableau 1 : Choix des cas

\begin{tabular}{|c|c|c|c|c|}
\hline Cas & Activité & Client & Evénement étudié & Entretiens \\
\hline 1. Mecano & $\begin{array}{l}\text { Sous-traitant de maintenance } \\
\text { (fournisseur en place) }\end{array}$ & Industrie chimique & $\begin{array}{l}\text { Réorganisation en vue d'un changement } \\
\text { de sous-traitant }\end{array}$ & Acheteur, responsable de maintenance \\
\hline 2. Maintini & $\begin{array}{l}\text { Sous-traitant de maintenance } \\
\text { (outsider) }\end{array}$ & $\begin{array}{l}\text { Industrie } \mathrm{de} \\
\text { transformation (verre) }\end{array}$ & Changement de sous-traitant & $\begin{array}{l}\text { Acheteur, responsable de la maintenance, } \\
\text { ingénieur commercial et dirigeant du nouveau } \\
\text { fournisseur }\end{array}$ \\
\hline 3. Informa & Achat de prestation informatique & Informatique & $\begin{array}{l}\text { Négociation annuelle, affectation des } \\
\text { projets }\end{array}$ & $\begin{array}{l}\text { Acheteur, client interne, commercial } \\
\text { prestataire }\end{array}$ \\
\hline 4. Color & $\begin{array}{l}\text { Fournisseur en peinture } \\
\text { (fournisseur en place) }\end{array}$ & Sous-traitant automobile & Changement de fournisseur & Acheteur, client interne \\
\hline 5. Electro & $\begin{array}{l}\text { Sous-traitant en composants } \\
\text { électroniques } \\
\text { (fournisseur en place) }\end{array}$ & $\begin{array}{l}\text { Fournisseur de systèmes } \\
\text { pour l'aéronautique }\end{array}$ & $\begin{array}{l}\text { Choix du sous-traitant pour chaque } \\
\text { nouveau composant parmi un ensemble } \\
\text { stable de partenaires }\end{array}$ & Acheteur, chef de projet client \\
\hline 6. Renovbati & $\begin{array}{l}\text { Sous-traitant de travaux de rénovation } \\
\text { d'un bâtiment industriel } \\
\text { (outsider) }\end{array}$ & Industrie mécanique & Choix des sous-traitants & $\begin{array}{l}\text { Acheteur, responsable travaux neufs, le chef } \\
\text { de projet côté fournisseur }\end{array}$ \\
\hline 7. Renovutil & $\begin{array}{l}\text { Sous-traitant pour la rénovation des } \\
\text { «utilités » } \\
\text { (outsider et insider selon les cas) }\end{array}$ & $\begin{array}{l}\text { Industrie } \\
\text { transformation } \\
\text { (matériaux) }\end{array}$ & $\begin{array}{l}\text { Achat ponctuel, choix d'une société } \\
\text { d'ingénierie et de travaux }\end{array}$ & $\begin{array}{l}\text { Acheteur et responsable travaux neufs de deux } \\
\text { clients différents, trois ingénieurs } \\
\text { commerciaux }\end{array}$ \\
\hline 8. Waste & $\begin{array}{l}\text { Prestataire de traitement des déchets } \\
\text { (nouveau contrat) }\end{array}$ & Industrie chimique & Achat d'une prestation & Acheteur, client interne \\
\hline
\end{tabular}


Tableau 2. : Comparaison des cas

\begin{tabular}{|c|c|c|c|c|c|c|c|c|c|}
\hline Cas & Activité & $\begin{array}{l}\text { Décalage entre } \\
\text { agendas }\end{array}$ & 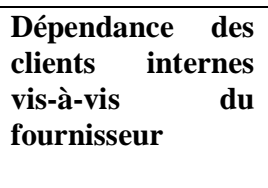 & $\begin{array}{l}\text { Attitude } r \\
\text { fournisseurs en place } \\
\text { et des nouveaux } \\
\text { fournisseurs }\end{array}$ & $\begin{array}{lr}\text { Actions } & \\
\text { engagées } & \text { pour } \\
\text { ouvrir } & \text { le } \\
\text { marché } & \end{array}$ & \begin{tabular}{lr}
\multicolumn{2}{l}{ Alignement } \\
agendas & et \\
intérêts & entre \\
acteurs & \\
internes & \\
\end{tabular} & $\begin{array}{l}\text { Niveau de } \\
\text { centralisation } \\
\text { des achats }\end{array}$ & $\begin{array}{l}\text { Relations entre } \\
\text { acteurs } \\
\text { internes }\end{array}$ & $\begin{array}{l}\text { Niveau d'ouverture } \\
\text { de la relation client } \\
\text { fournisseur }\end{array}$ \\
\hline 1. Mecano & $\begin{array}{ll}\text { Changement } & \mathrm{de} \\
\text { sous-traitant } & \mathrm{de} \\
\text { maintenance } & \end{array}$ & Politique achat & $\begin{array}{l}\text { Coproduction } \\
\text { significative }\end{array}$ & $\begin{array}{l}\text { En place : renforcer la } \\
\text { dépendance } \\
\text { Outsider : inexistant }\end{array}$ & $\begin{array}{l}\text { Rationalisation } \\
\text { de l'activité }\end{array}$ & Très faible & $\begin{array}{l}\text { Fonction Achats } \\
\text { centralisée et } \\
\text { distante }\end{array}$ & $\begin{array}{l}\text { Conflit, puis, } \\
\text { accord tacite } \\
\text { d'évitement }\end{array}$ & $\begin{array}{l}\text { Maintien d'une } \\
\text { relation exclusive } \\
\text { avec le prestataire en } \\
\text { place }\end{array}$ \\
\hline $\begin{array}{l}2 . \\
\text { Maintini }\end{array}$ & $\begin{array}{ll}\text { Changement } & \mathrm{de} \\
\text { sous-traitant } & \mathrm{de} \\
\text { maintenance } & \end{array}$ & $\begin{array}{l}\text { Demande } \\
\text { client interne }\end{array}$ & $\begin{array}{l}\text { Coproduction } \\
\text { significative } \\
\text { Coprescription } \\
\text { significative }\end{array}$ & 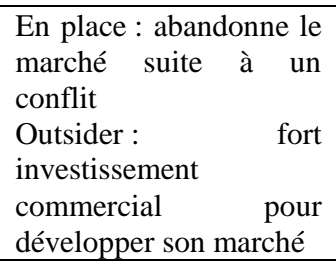 & $\begin{array}{l}\text { Coprescription } \\
\text { ouverte, } \\
\text { formalisation } \\
\text { des règles de la } \\
\text { coproduction }\end{array}$ & $\begin{array}{l}\text { Forte autonomie } \\
\text { budgétaire de } \\
\text { l'usine }\end{array}$ & $\begin{array}{l}\text { Fonction Achats } \\
\text { centralisée et } \\
\text { distante }\end{array}$ & $\begin{array}{l}\text { Accord sur une } \\
\text { autonomie du } \\
\text { client interne } \\
\text { dans le choix }\end{array}$ & $\begin{array}{l}\text { Ouverture limitée à } \\
\text { un seul outsider, prix } \\
\text { conventionnel }\end{array}$ \\
\hline 3. Informa & $\begin{array}{ll}\text { Achat } & \text { de } \\
\text { prestation } & \\
\text { informatique } & \end{array}$ & \begin{tabular}{l}
\multicolumn{2}{l}{ Renouvellement } \\
annuel des \\
contrats
\end{tabular} & $\begin{array}{l}\text { Coproduction très } \\
\text { significative } \\
\text { Coprescription très } \\
\text { significative }\end{array}$ & $\begin{array}{l}\text { Plusieurs fournisseurs } \\
\text { en place, en } \\
\text { compétition. Essaient } \\
\text { de se placer le plus en } \\
\text { amont lors des } \\
\text { nouveaux projets } \\
\text { Outsider : inexistants }\end{array}$ & $\begin{array}{l}\text { Coprescription } \\
\text { fermée } \\
\text { Rationalisation } \\
\text { de l'activité }\end{array}$ & $\begin{array}{l}\text { Forte autonomie } \\
\text { budgétaire }\end{array}$ & $\begin{array}{l}\text { Fonction Achats } \\
\text { centralisée et } \\
\text { distante }\end{array}$ & $\begin{array}{l}\text { Accord tacite } \\
\text { d'évitement }\end{array}$ & $\begin{array}{l}\text { Un club fermé de } \\
\text { prestataire } \\
\text { «insiders », des } \\
\text { relations étroites avec } \\
\text { les clients internes, } \\
\text { prix conventionnel }\end{array}$ \\
\hline 4. Color & $\begin{array}{ll}\text { Changement } & \text { de } \\
\text { fournisseurs } & \text { en } \\
\text { peinture } & \end{array}$ & $\begin{array}{l}\text { Politique achat, } \\
\text { changement } \\
\text { imposé par la } \\
\text { direction des } \\
\text { achats }\end{array}$ & $\begin{array}{l}\text { Coproduction } \\
\text { significative avec } \\
\text { le fournisseur en } \\
\text { place }\end{array}$ & $\begin{array}{l}\text { Fournisseur en place: } \\
\text { renforcer la dépendance } \\
\text { Nouveau fournisseur } \\
\text { déjà présent dans une } \\
\text { autre usine : recherche } \\
\text { de volume }\end{array}$ & $\begin{array}{l}\text { Standardisation } \\
\text { de l'activité } \\
\text { Coprescription } \\
\text { avec le nouveau } \\
\text { fournisseur }\end{array}$ & $\begin{array}{lr}\text { Décision } & \text { prise } \\
\text { au niveau de la } \\
\text { Business } & \text { Units, } \\
\text { pas } & \text { de } \\
\text { cohérence } & \\
\text { budgétaire } & \text { au } \\
\text { niveau } & \text { de } \\
\text { l'usine } & \end{array}$ & $\begin{array}{l}\text { Fonction Achats } \\
\text { centralisée } \\
\text { (niveau BU), } \\
\text { fortement } \\
\text { interventionniste }\end{array}$ & $\begin{array}{l}\text { Conflit entre } \\
\text { achat (niveau } \\
\text { BU) et usine } \\
\text { puis relation de } \\
\text { coopération }\end{array}$ & $\begin{array}{l}\text { Ouverture } \\
\text { significative, } \\
\text { changement } \\
\text { fournisseur, } \\
\text { baisse du prix }\end{array}$ \\
\hline
\end{tabular}




\begin{tabular}{|c|c|c|c|c|c|c|c|c|c|}
\hline Cas & Activité & $\begin{array}{l}\text { Décalage entre } \\
\text { agendas }\end{array}$ & $\begin{array}{l}\text { Dépendance } \\
\text { clients internes } \\
\text { vis-à-vis } \\
\text { fournisseur }\end{array}$ & $\begin{array}{l}\text { Attitude } \\
\text { fournisseurs en place } \\
\text { et des nouveaux } \\
\text { fournisseurs }\end{array}$ & $\begin{array}{lr}\text { Actions } & \\
\text { engagées } & \text { pour } \\
\text { ouvrir } & \text { le } \\
\text { marché } & \end{array}$ & \begin{tabular}{lr}
\multicolumn{2}{l}{ Alignement des } \\
agendas & et \\
intérêts & entre \\
acteurs & \\
internes & \\
\end{tabular} & $\begin{array}{l}\text { Niveau de } \\
\text { centralisation } \\
\text { des achats }\end{array}$ & $\begin{array}{l}\text { Relations entre } \\
\text { acteurs } \\
\text { internes }\end{array}$ & $\begin{array}{l}\text { Niveau d'ouverture } \\
\text { de la relation client } \\
\text { fournisseur }\end{array}$ \\
\hline 5. Electro & $\begin{array}{ll}\text { Mise } & \text { en } \\
\text { concurrence } & \text { de } \\
\text { sous-traitants en } & \text { en } \\
\text { composants } & \\
\text { électroniques }\end{array}$ & $\begin{array}{l}\text { A la demande } \\
\text { d'une partie des } \\
\text { clients internes }\end{array}$ & $\begin{array}{l}\text { Coprescription } \\
\text { significative } \\
\text { (réalisation de } \\
\text { prototypes) } \\
\text { Coproduction } \\
\text { limitée }\end{array}$ & $\begin{array}{l}\text { à } 5 \text { fournisseurs en } \\
\text { place : maintenir le } \\
\text { client dépendant en } \\
\text { investissant en amont } \\
\text { dans les prototypes, }\end{array}$ & $\begin{array}{l}\text { Sélection et } \\
\text { définition du } \\
\text { prix en amont } \\
\text { du processus de } \\
\text { coprescription }\end{array}$ & \begin{tabular}{l}
\multicolumn{2}{l}{ Faible incitation } \\
budgétaire au \\
niveau $\quad$ de \\
l'équipe de \\
développement
\end{tabular} & $\begin{array}{l}\text { Fonction Achats } \\
\text { centralisée, } \\
\text { fortement } \\
\text { interventionniste }\end{array}$ & $\begin{array}{lr}\text { Conflit entre } \\
\text { acheteur } & \text { et } \\
\text { concepteurs puis } \\
\text { relation r de } \\
\text { coopération }\end{array}$ & $\begin{array}{l}\text { Compétition en } \\
\text { amont du processus } \\
\text { de développement, au } \\
\text { sein d'un club fermé } \\
\text { de fournisseurs, prix } \\
\text { négocié }\end{array}$ \\
\hline $\begin{array}{l}6 . \\
\text { Renovbati }\end{array}$ & $\begin{array}{ll}\text { Mise } & \text { en } \\
\text { concurrence } & \text { de } \\
\text { prestataires de } & \text { de } \\
\text { travaux pour la } \\
\text { rénovation d'un } \\
\text { bâtiment } \\
\text { industriel }\end{array}$ & $\begin{array}{l}\text { Nouvelle } \\
\text { politique achat, } \\
\text { rôle croissant de } \\
\text { l'achat }\end{array}$ & $\begin{array}{l}\text { Coprescription très } \\
\text { significative } \\
\text { Coproduction } \\
\text { significative }\end{array}$ & $\begin{array}{l}\text { En place : accompagner } \\
\text { le client interne, } \\
\text { développer la confiance } \\
\text { Outsider: } \\
\text { investissement variable }\end{array}$ & $\begin{array}{l}\text { Coprescription } \\
\text { ouverte }\end{array}$ & $\begin{array}{l}\text { Faible } \\
\text { autonomie } \\
\text { budgétaire } \\
\text { directeur } \\
\text { travaux }\end{array}$ & $\begin{array}{l}\text { Fonction } \\
\text { présente suats } \\
\text { l'usine }\end{array}$ & $\begin{array}{l}\text { Relation de } \\
\text { coopération sur } \\
\text { certains lots, } \\
\text { évitement sur les } \\
\text { autres }\end{array}$ & $\begin{array}{l}\text { Ouverture partielle } \\
\text { du marché } r \text { sur } \\
\text { certains lots, prix } \\
\text { négocié }\end{array}$ \\
\hline $\begin{array}{l}7 . \\
\text { Renovutil }\end{array}$ & $\begin{array}{l}\text { Prestataires de } \\
\text { travaux, pour la } \\
\text { rénovation des } \\
\text { utilités }\end{array}$ & $\begin{array}{l}\text { Clients internes } \\
\text { dépourvus de } \\
\text { solutions }\end{array}$ & $\begin{array}{l}\text { Coprescription } \\
\text { significative } \\
\text { Coproduction } \\
\text { limitée }\end{array}$ & $\begin{array}{l}\text { En place : contribution } \\
\text { à la prescription } \\
\text { Outsider : } \\
\text { investissement } \\
\text { significatif pour prendre } \\
\text { place }\end{array}$ & $\begin{array}{l}\text { Coprescription } \\
\text { ouverte } \\
(2 \text { prestataires } \\
\text { en concurrence })\end{array}$ & $\begin{array}{l}\text { Usine autonome } \\
\text { sur son budget } \\
\text { d'investissement }\end{array}$ & $\begin{array}{l}\text { Fonction Achats } \\
\text { centralisée }\end{array}$ & $\begin{array}{ll}\text { Relation } & \text { de } \\
\text { coopération } & \end{array}$ & $\begin{array}{l}\text { Ouverture } \\
\text { significative, } \\
\text { introduction d'un } \\
\text { nouveau prestataire } \\
\text { dans un cas, échec } \\
\text { dans le second, prix } \\
\text { négocié }\end{array}$ \\
\hline 8. Waste & $\begin{array}{l}\text { Achat d'une } \\
\text { prestation } \\
\text { spécialisée de } \\
\text { traitement des } \\
\text { déchets }\end{array}$ & $\begin{array}{l}\text { Prestation } \\
\text { ponctuelle }\end{array}$ & $\begin{array}{l}\text { Coprescription } \\
\text { significative } \\
\text { Pas de } \\
\text { coproduction }\end{array}$ & $\begin{array}{llr}\text { En place : } & \text { forte } \\
\text { contribution à } & \text { la } \\
\text { prescription } & & \\
\text { Outsider : inexistant } & \end{array}$ & $\begin{array}{l}\text { Coprescription } \\
\text { fermée }\end{array}$ & $\begin{array}{l}\text { Faible } \\
\text { autonomie } \\
\text { budgétaire }\end{array}$ & $\begin{array}{l}\text { Fonction } \\
\text { présente } \\
\text { l'usine }\end{array}$ & $\begin{array}{lr}\text { Stratégie } & \text { de } \\
\text { blocage } & \text { de } \\
\text { l'acheteur } & \text { puis } \\
\text { arbitrage } & \text { du } \\
\text { directeur } & \\
\text { d'usine } & \\
\end{array}$ & $\begin{array}{l}\text { Relation exclusive, } \\
\text { prix imposé par le } \\
\text { prestataire }\end{array}$ \\
\hline
\end{tabular}




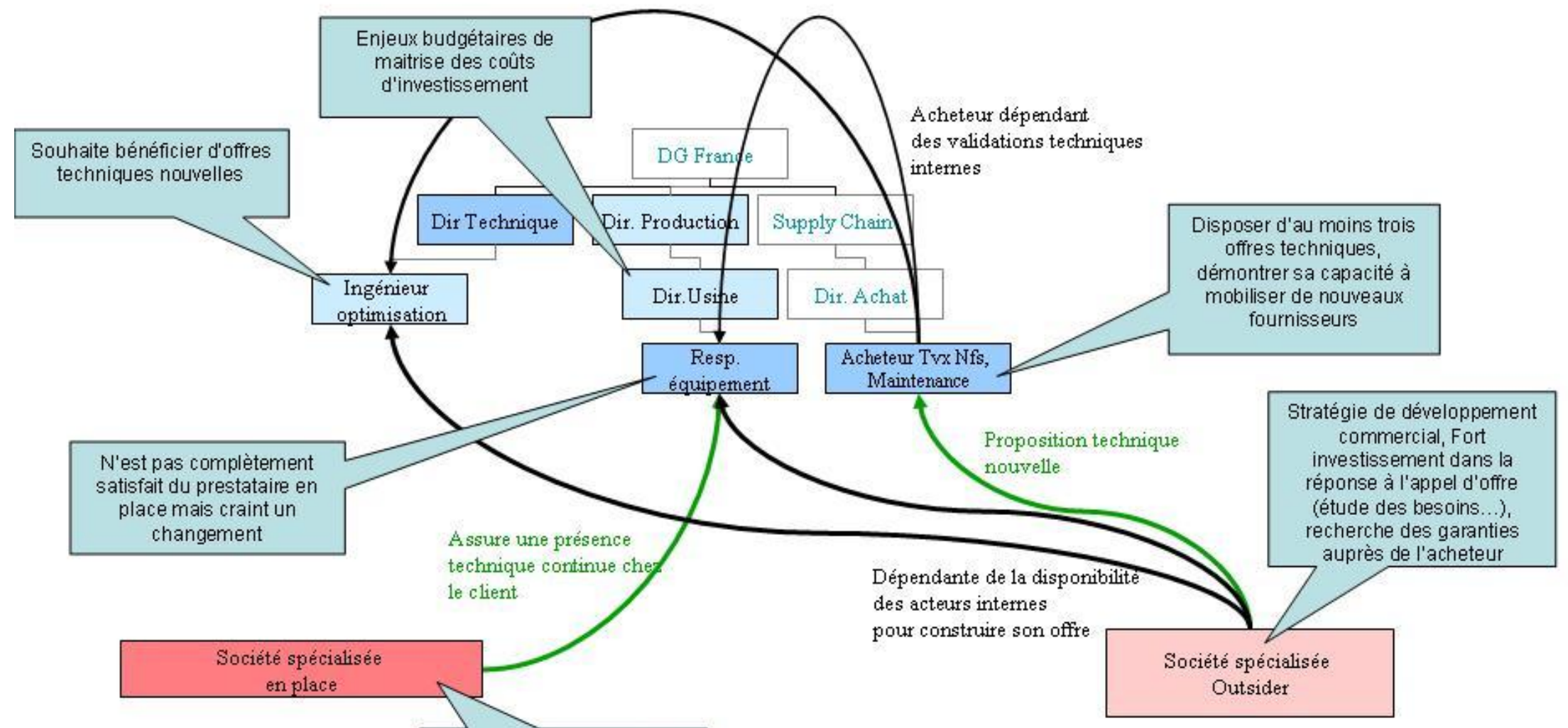

Cas Renovutil (1) 


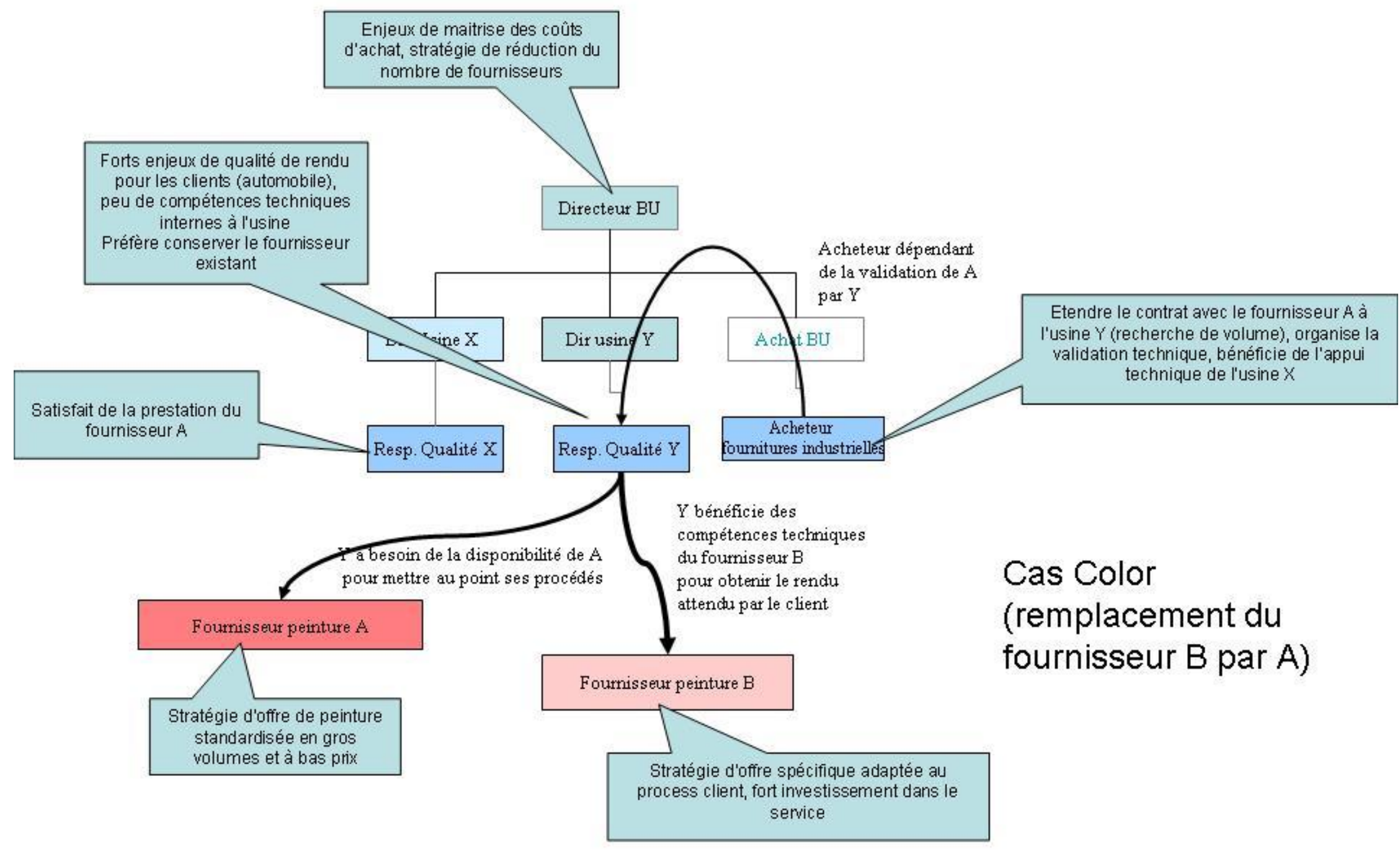

